\title{
Polyhedral models for generalized associahedra via Coxeter elements
}

\author{
Salvatore Stella
}

Received: 7 December 2011 / Accepted: 31 August 2012 / Published online: 22 September 2012

(C) Springer Science+Business Media, LLC 2012

\begin{abstract}
Motivated by the theory of cluster algebras, F. Chapoton, S. Fomin, and A. Zelevinsky associated to each finite type root system a simple convex polytope, called generalized associahedron. They provided an explicit realization of this polytope associated with a bipartite orientation of the corresponding Dynkin diagram.

In the first part of this paper, using the parametrization of cluster variables by their $g$-vectors explicitly computed by S.-W. Yang and A. Zelevinsky, we generalize the original construction to any orientation. In the second part we show that our construction agrees with the one given by $\mathrm{C}$. Hohlweg, C. Lange, and H. Thomas in the setup of Cambrian fans developed by N. Reading and D. Speyer.
\end{abstract}

Keywords Generalized associahedra · Cluster algebras · Cluster complexes · $g$-vectors $\cdot c$-sortability $\cdot$ Cambrian fans

\section{Introduction}

Much information on the structure of a cluster algebra $\mathcal{A}$ can be deduced directly from a purely combinatorial gadget: its cluster complex. It is an abstract simplicial complex whose vertices are the cluster variables of $\mathcal{A}$ and whose maximal simplices are given by clusters. In this paper we restrict our attention to finite type cluster algebras; under such assumption the cluster complex is finite.

A complete classification of finite type cluster algebras was given in [5]: it is identical to the Cartan-Killing classification of semisimple Lie algebras and crystallographic root systems. In the same paper, under the assumption that the initial cluster is bipartite, Fomin and Zelevinsky provided an explicit combinatorial description of

S. Stella $(\bowtie)$

Department of Mathematics, Northeastern University, 360 Huntington ave, 567 Lake Hall, Boston, MA 02115, USA

e-mail: stella.sa@husky.neu.edu 
the cluster complex obtained by labeling its vertices with almost-positive roots in the corresponding root system.

They constructed a function on ordered pairs of labels, called compatibility degree, encoding whether the corresponding cluster variables are compatible (i.e. they belong to the same cluster), exchangeable or neither. Its definition is purely combinatorial and does not refer to the cluster algebra but just to the labels. The description of the cluster complex they presented is in terms of this function: compatible pairs of almost-positive roots form its 1-skeleton; higher dimension simplices are given by the cliques of the 1 -skeleton.

In [6] the authors improved on this combinatorial model explaining how almostpositive roots give a geometric realization of the cluster complex. They showed that the positive real span of the labels in any simplex of the cluster complex is a cone in a complete simplicial fan: the cluster fan. Among the applications of this realization there is a parametrization of cluster monomials in $\mathcal{A}$ with points of the root lattice $Q$ and an explicit formula for all the exchange relations in the coefficient-free case.

Further study ([3]) of the cluster fan showed that it is the normal fan of a distinguished polytope: the generalized associahedron of the given type. Its description is completely explicit: the authors discussed all the constrains that its support function must satisfy and then provided a concrete function that meets them.

As noted above the construction in [6] and [3] depends on the labeling of cluster variables of $\mathcal{A}$ by almost-positive roots; such a parametrization is provided by their denominator vectors with respect to a bipartite initial cluster. Using the notion of g-vectors from [7], in [18] Yang and Zelevinsky generalized this parametrization of cluster variables to a family of parametrizations, one for each acyclic initial cluster, by a subset $\Pi(c)$ of the associated weight lattice (as is customary we use a Coxeter element $c$ in the Weyl group to keep track of the orientation of the initial cluster; see (1) and (2) for details on the conventions we adopt).

The first goal of this paper is to extend the results from [6] and [3] to each of these new parametrizations. Retracing the steps in those papers, for any choice of acyclic initial cluster, we will construct a complete simplicial fan realizing the cluster complex and we will show that it is the normal fan to a geometric realization of a generalized associahedron. We can summarize our claims as follows:

Theorem 1 Let $\mathcal{A}$ be a cluster algebra of finite type with an acyclic initial cluster and let $c$ be the Coxeter element encoding the initial orientation. Let $\Pi(c)$ be the labeling set and $(\bullet \| \bullet)_{c}$ its compatibility degree function both constructed in [18]. Then

1. Every c-cluster in $\Pi(c)$ (i.e. every maximal subset of $\Pi(c)$ consisting of pairwise compatible weights) is a $\mathbb{Z}$-basis of the weight lattice $P$.

2. The positive linear spans of the simplices in the clique complex induced by $(\bullet \| \bullet)_{c}$ on $\Pi(c)$ form a complete simplicial fan $\mathcal{F}_{c}^{\Pi}$ realizing the cluster complex. Cluster monomials of $\mathcal{A}$ are in bijection with points of $P$.

3. $\mathcal{F}_{c}^{\Pi}$ is the normal fan to a simple polytope: a geometric realization of the associated generalized associahedron.

4. If $\mathcal{A}$ is coefficient-free then all its exchange relations are explicitly determined by the labels of exchangeable cluster variables. 
The proof will be split into sub-statements, namely Theorems 2, 3, 4, and 5. Some of these results were already proved in less generality or were already conjectured; we will provide explicit references in Sect. 2.

It turns out that our polytopes are the same as those studied in [11] in the setup of Cambrian fans developed by Reading and Speyer. The construction we propose, however, is different from the one by Hohlweg, Lange, and Thomas. This provides us with an alternative prospective on $c$-cluster combinatorics that allows us to recover all the exchange relations of the associated coefficient-free cluster algebra and to answer positively to Problem 4.1 posed in [10].

To explain what we mean by "different" recall that the definition of Cambrian fans is given in terms of its maximal cones as opposed to the definition of cluster fans that builds up from the 1-skeleton. Indeed to each Coxeter element $c$ of a finite type Weyl group $W$ one can associate a lattice congruence on the group itself (seen as a lattice for the right weak order). This produces a coarsening of the associated Coxeter fan obtained by glueing together cones corresponding to elements in the same class (recall that the Coxeter fan is the complete simplicial fan in the weight space of $W$ whose maximal cones are the images of the fundamental Weyl chamber under the action of the group). The approach used in [11] to show that the Cambrian fans are polytopal follows the same philosophy: they begin from the generalized permutahedron associated to $W$ seen as intersection of half-spaces and, again using the lattice congruence induced by $c$, they remove a certain subset of them to make it into a generalized associahedron.

The second goal of this paper is to show that the generalizations of the cluster fans we propose coincide with the Cambrian fans of Reading and Speyer. To do so it suffices to show that the polyhedral models for the generalized associahedra we build are the same as the realizations given in [11]. Note that in type $A$ the interaction between the geometric realizations of the associahedron by Hohlweg, Lange, and Thomas and the original realization by Chapoton, Fomin, and Zelevinsky has been already investigated in [2].

The paper is structured as follows: in Sect. 2, after having recalled the required terminology and having set up some notations, we discuss in more detail our generalizations of the results in [6] and [3] and we provide an idea of the strategy we adopt to prove them. We then recall some more terminology and explain how our construction relates to Cambrian fans and to the polytopes from [11].

In Sect. 3 we introduce the main tool of the paper: the set of c-almost-positive roots $\Phi_{\text {ap }}(c)$. Many arguments from [6] and [3] require to perform an induction on the rank of the cluster algebra; the labeling of cluster variables by almost-positive roots is ideal for such a purpose. In our case, however, we are given a set of weights to parametrize the vertices of the cluster complex therefore we cannot generalize those proofs directly. The solution we adopt is to identify the weight lattice with the root lattice in such a way that the restriction to a smaller rank cluster sub-algebra can be expressed easily in terms of the labels in a new set $\Phi_{\text {ap }}(c)$ (the image of $\Pi(c)$ under this identification).

Section 4 deals with bipartite orientations. We show that, in this case, our results follow directly from their analogues from [6] and [3].

Section 5 contains the proofs of some technical results we need in Sect. 6 where we complete the proofs of the main results of the first part of the paper. 
The paper is concluded by Sect. 7 where we show that our realizations of the generalized associahedra coincide with those constructed by Hohlweg, Lange, and Thomas and therefore that our generalization of cluster fans is a different presentation of Cambrian fans.

\section{Preliminaries}

We start by setting up notation and recalling some terminology and results from [18]. Let $I$ be a finite type Dynkin diagram; with a small abuse of notation denote by $I$ also its vertex set. Let $W$ be the associated Weyl group with simple reflections $\left\{s_{i}\right\}_{i \in I}$ and let $A=\left(a_{i j}\right)_{i, j \in I}$ be the corresponding Cartan matrix.

Recall that an element $c$ of $W$ is said to be Coxeter if every simple reflection appears in a reduced expression of $c$ exactly once. To each Coxeter element $c$ associate a skew-symmetrizable matrix $B(c)=\left(b_{i j}\right)_{i, j \in I}$ as follows. For $i$ and $j$ in $I$, write $i \prec_{c} j$ if $i$ and $j$ are connected by an edge and $s_{i}$ precedes $s_{j}$ in a reduced expression of $c$. Set then

$$
b_{i j}:= \begin{cases}-a_{i j} & \text { if } i \prec_{c} j \\ a_{i j} & \text { if } j \prec_{c} i \\ 0 & \text { otherwise. }\end{cases}
$$

Note also that Coxeter elements are in bijection with orientation of $I$ under the convention

$$
j \rightarrow i \quad \Leftrightarrow \quad i \prec_{c} j
$$

Remark 1 In each Weyl group there is a distinguished class of Coxeter elements (call them bipartite) corresponding to orientations of $I$ in which each node is either a source or a sink. Following the notation of [6], we denote bipartite Coxeter elements by $t$.

For a given Coxeter element $c$ denote by $\mathcal{A}_{0}(c)$ the coefficient-free cluster algebra with the initial $B$-matrix $B(c)$. Let $\left\{\omega_{i}\right\}_{i \in I}$ be the set of fundamental weights associated to $I$ and $w_{0}$ the longest element in $W$. Set $h(i ; c)$ to be the minimum positive integer such that

$$
c^{h(i ; c)} \omega_{i}=-\omega_{i}{ }^{*}
$$

where $\omega_{i *}:=-w_{0} \omega_{i}$ (cf. Proposition 1.3 in [18]).

By Theorem 1.4 in [18] the set of weights

$$
\Pi(c):=\left\{c^{m} \omega_{i}: i \in I, 0 \leq m \leq h(i ; c)\right\}
$$

parametrizes the cluster variables in $\mathcal{A}_{0}(c)$. The correspondence is given associating to each cluster variable its $g$-vector as defined in [7]; in particular cluster variables in the initial cluster correspond to fundamental weights.

The set $\Pi(c)$ can be made into an abstract simplicial complex of pure dimension $n-1$ (the $c$-cluster complex) as follows. The cluster algebra structure induces a 
permutation on $\Pi(c)$

$$
\tau_{c}^{\Pi}(\lambda):= \begin{cases}\omega_{i} & \text { if } \lambda=-\omega_{i} \\ c \lambda & \text { otherwise }\end{cases}
$$

and a (unique) $\tau_{c}^{\Pi}$-invariant $c$-compatibility degree function defined by the initial conditions

$$
\left(\omega_{i} \| \lambda\right)_{c}^{\Pi}:=\left[\left(c^{-1}-1\right) \lambda ; \alpha_{i}\right]_{+}
$$

where $\left[\bullet ; \alpha_{i}\right]$ is the coefficient of $\alpha_{i}$ in $\bullet$ expressed in the basis of simple roots and $[\bullet]_{+}$denotes $\max \{\bullet, 0\}$ (cf. Proposition 5.1 in [18]).

Note that the action of $\tau_{c}^{\Pi}$ on $\Pi(c)$ is, by construction, compatible with the action of $w_{0}$ on $I$; that is any $\tau_{c}^{\Pi}$-orbit contains a unique pair $\left\{\omega_{i}, \omega_{i *}\right\}$ (or a single fundamental weight $\omega_{i}$ if $\left.i=i^{*}\right)$.

Call two weights $\lambda$ and $\mu$ in $\Pi(c) c$-compatible if

$$
(\lambda \| \mu)_{c}^{\Pi}=0 .
$$

This definition makes sense since the $c$-compatibility degree satisfies

$$
(\lambda|| \mu)_{c}^{\Pi}=0 \Leftrightarrow(\mu \| \lambda)_{c}^{\Pi}=0 .
$$

The $c$-cluster complex $\Delta_{c}^{\Pi}$ is defined to be the abstract simplicial complex on the vertex set $\Pi(c)$ whose 1 -skeleton is given by $c$-compatible pairs of weights and whose higher dimensional simplex are given by the cliques of its 1-skeleton. We refer to its maximal simplices as c-clusters; this name already appeared in the work of Reading and Speyer in a different setup, we will discuss later on how the two notions are related.

The first step in order to construct a complete simplicial fan realizing the $c$-cluster complex is to show that we can associate an $n$-dimensional cone to each $c$-cluster.

Theorem 2 Each c-cluster in $\Delta_{c}^{\Pi}$ is a $\mathbb{Z}$-basis of the weight lattice $P$.

Remark 2 Theorem 2 was conjectured in [7] (Conjecture 7.10(2)) and then proved in [4] (Theorem 1.7) under the assumption that the initial exchange matrix is skewsymmetric.

Let $\mathcal{F}_{c}^{\Pi}$ be the collection of all the cones in $P_{\mathbb{R}}$ that are positive linear span of simplices in the $c$-cluster complex.

Theorem $3 \mathcal{F}_{c}^{\Pi}$ is a complete simplicial fan.

Remark 3 This is a generalization of Theorem 1.10 in [6], and our proof is inspired by the one in that paper. In particular we will deduce the result from the following proposition (mimicking Theorem 3.11 in there). 
Proposition 1 Every point $\mu$ in the weight lattice $P$ can be uniquely be written as

$$
\mu=\sum_{\lambda \in \Pi(c)} m_{\lambda} \lambda
$$

where all the coefficients $m_{\lambda}$ are non-negative integers and $m_{\lambda} m_{v}=0$ whenever $(\lambda \| v)_{c}^{\Pi} \neq 0$

The expression (3) is called the $c$-cluster expansion of $\mu$.

A simplicial fan is said to be polytopal if it is the normal fan to a simple polytope. Recall that, given a simple full-dimensional polytope $T$ in a vector space $V$, its support function $F$ is the piecewise-linear function on $V^{*}$ defined by

$$
\begin{aligned}
F: V^{*} & \longrightarrow \mathbb{R} \\
\varphi & \longmapsto \max \{\varphi(x) \mid x \in T\}
\end{aligned}
$$

and its normal fan is the complete simplicial fan in $V^{*}$ whose maximal cones are the domains of linearity of $F$. Note that in dimension greater than 2 not every simplicial fan needs to be the normal fan of a polytope (see for example Sect. 1.5 in [8]).

Our next goal is to show that the $c$-cluster fans we constructed so far are polytopal. In view of Theorem 3 , each function defined on $\Pi(c)$ extends uniquely to a continuous, piecewise-linear function on $P_{\mathbb{R}}$ linear on the maximal cones of $\mathcal{F}_{c}^{\Pi}$. In particular, every function

$$
f: I \longrightarrow \mathbb{R}
$$

satisfying $f(i)=f\left(i^{*}\right)$ gives rise to a continuous, $\tau_{c}^{\Pi}$-invariant, piecewise-linear function $F_{c}=F_{c ; f}$, by setting

$$
F_{c}\left(c^{m} \omega_{i}\right):=f(i)
$$

for all $c^{m} \omega_{i} \in \Pi(c)$, and then extending it to $P_{\mathbb{R}}$ as above.

Let $\operatorname{Asso}_{c}^{f}(W)$ be the subset of $P_{\mathbb{R}}^{*}$ defined by

$$
\operatorname{Asso}_{c}^{f}(W):=\left\{\varphi \in P_{\mathbb{R}}^{*} \mid \varphi(\lambda) \leq F_{c}(\lambda), \forall \lambda \in \Pi(c)\right\} .
$$

Theorem 4 If $f: I \rightarrow \mathbb{R}$ is such that

1. for any $i \in I$

$$
f(i)=f\left(i^{*}\right)
$$

2. for any $j \in I$

$$
\sum_{i \in I} a_{i j} f(i)>0
$$

then $\operatorname{Asso}_{c}^{f}(W)$ is a simple $n$-dimensional polytope with support function $F_{c}$. Furthermore, the domains of linearity of $F_{c}$ are exactly the maximal cones of $\mathcal{F}_{c}^{\Pi}$, hence the normal fan of $\operatorname{Asso}_{c}^{f}(W)$ is $\mathcal{F}_{c}^{\Pi}$. 
Remark 4 Theorem 4 is a generalization of Theorem 1.5 in [3]. Its proof uses the result by Chapoton, Fomin, and Zelevinsky as base case.

The following examples illustrate the above results. We represent a point $\varphi \in P_{\mathbb{R}}^{*}$ by a tuple $\left(z_{i}:=\varphi\left(\omega_{i}\right)\right)_{i \in I}$. We also use the standard numeration of simple roots and fundamental weights from [1].

The construction carried on in this paper, as it will be explained in detail in Sect. 4, coincides with the one in [6] and [3] when $c$ is a bipartite Coxeter element. Therefore the first example in which something interesting arises is $c=s_{1} s_{2} s_{3}$ in type $A_{3}$. In this case $\Pi(c)$ consists of two $\tau_{c}^{\Pi}$-orbits:

$$
\omega_{1} \stackrel{\tau_{c}}{\rightleftarrows}-\omega_{1}+\omega_{2} \stackrel{\tau_{c}}{\longrightarrow}-\omega_{2}+\omega_{3} \stackrel{\tau_{c}}{\longrightarrow}-\omega_{3} \stackrel{\tau_{c}}{\longrightarrow} \omega_{3} \stackrel{\tau_{c}}{\longrightarrow}-\omega_{1}
$$

and

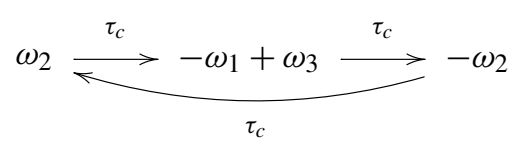

It is not surprising that the number of orbits and their lengths are the same as the $A_{3}$ example in [3]: they depend only on the type of the cluster algebra and not on the choice of a Coxeter element. Since in this case $w_{0} \omega_{1}=-\omega_{3}$ we have $1^{*}=3$ therefore we need to impose $f(1)=f(3)$; condition (2) in Theorem 4 becomes

$$
0<f(1)<f(2)<2 f(1)
$$

and the corresponding polytope $\operatorname{Asso}_{c}^{f}(W)$ is defined by the inequalities

$$
\begin{array}{r}
\max \left\{z_{1},-z_{1}+z_{2},-z_{2}+z_{3},-z_{3}, z_{3},-z_{1}\right\} \leq f(1) \\
\max \left\{z_{2},-z_{1}+z_{3},-z_{2}\right\} \leq f(2) .
\end{array}
$$

This polytope is shown in Fig. 1. Note that, to make pictures easier to plot and view, the angles between fundamental weights are not drawn to scale, and each facet is labeled by the weight it is orthogonal to.

Now let $c=s_{1} s_{2} s_{3}$ in type $C_{3}$. Then the set $\Pi(c)$ consists of three orbits:

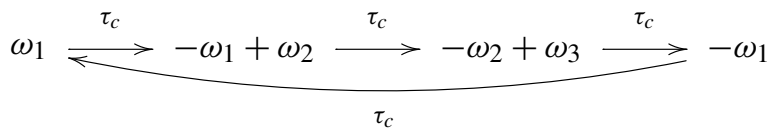

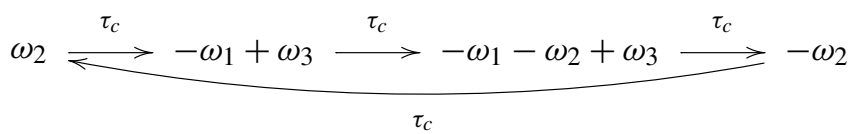




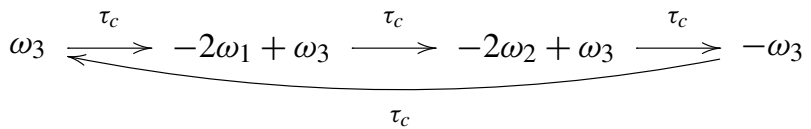

Condition (2) in Theorem 4 reads

$$
\begin{aligned}
f(2) & <2 f(1) \\
f(1)+f(3) & <2 f(2) \\
f(2) & <f(3)
\end{aligned}
$$

as in the corresponding example in [3]. The polytope is given by the inequalities

$$
\begin{array}{r}
\max \left\{z_{1},-z_{1}+z_{2},-z_{2}+z_{3},-z_{1}\right\}<f(1) \\
\max \left\{z_{2},-z_{1}+z_{3},-z_{1}-z_{2}+z_{3},-z_{2}\right\}<f(2) \\
\max \left\{z_{3},-2 z_{1}+z_{3},-2 z_{2}+z_{3},-z_{3}\right\}<f(3)
\end{array}
$$

and it is shown in Fig. 2 using the same conventions of Fig. 1.

To prove the results we discussed so far will use two types of argument. The first one is induction on the rank of $I$. Unfortunately the set $\Pi(c)$, and in general the whole weight lattice $P$, does not behave nicely when considering sub-diagrams of $I$. It is then convenient to introduce an auxiliary set of labels: the $c$-almost-positive roots:

$$
\Phi_{\mathrm{ap}}(c):=\left(c^{-1}-1\right) \Pi(c)
$$

whose behavior is more manageable. On the one hand the new set is related to the old one by a linear transformation therefore any property proved for $\Phi_{\text {ap }}(c)$ can be transported back to $\Pi(c)$.

On the other hand $\Phi_{\text {ap }}(c)$ is modeled after the set $\Phi_{\geq-1}$ introduced in [6]. It differs from the latter in several respects: first it still consists of $g$-vectors (in an odd-looking

Fig. $1 \operatorname{Asso}_{c}^{f}(W)$ in type $A_{3}$ for $c=s_{1} s_{2} s_{3}$

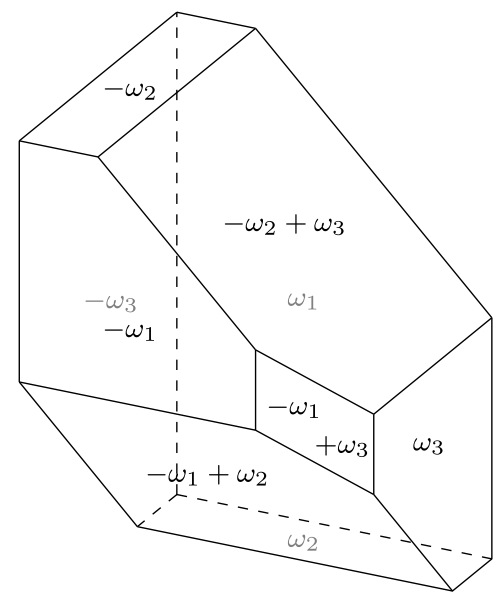


Fig. $2 \operatorname{Asso}_{c}^{f}(W)$ in type $C_{3}$ for $c=s_{1} s_{2} s_{3}$

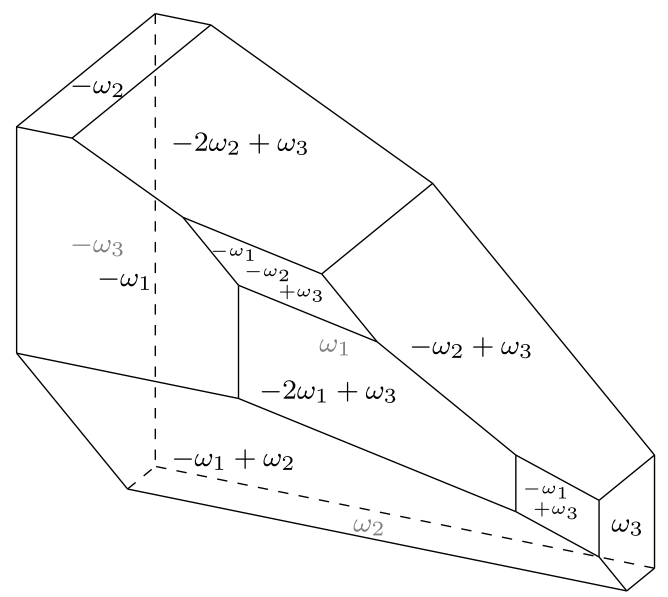

basis) and not denominator vectors; second it contains all the positive roots (as $\Phi_{\geq-1}$ does) but the negative simples are replaced by other negative roots depending on the choice of the Coxeter element $c$. However, contrary to what happens for $\Pi(c)$, it retains a notion of subset corresponding to a Dynkin sub-diagram. In order to use induction on $|I|$ it will then suffice to show that the $c$-compatibility degree on $\Phi_{\text {ap }}(c)$ is preserved when restricting to a sub-diagram of $I$ (this is the content of Proposition 5).

To explain the second type of argument we need an observation on Coxeter elements. For a given Coxeter element $c$, we call a simple reflection $s_{i}$ initial (resp. final) if $c$ admits a reduced expression of the form $c=s_{i} v$ (resp. $c=v s_{i}$ ). Conjugating any Coxeter element by an initial or final reflection produces another Coxeter element; call such a conjugation an elementary move and call two Coxeter elements related by a single elementary move adjacent. The following is a well-known fact.

Lemma 1 Any Coxeter element can be reached from any other via a sequence of elementary moves.

A proof can be found in [9] Theorem 3.1.4.

We will construct maps $\sigma_{i}^{ \pm 1}$ relating sets of $c$-almost-positive roots for adjacent Coxeter elements. These maps will not be linear so, a priori, they might not preserve all the properties we are interested into. Our strategy will be to show that, for any Coxeter element $c$, there exist a bipartite Coxeter element $t$ and a sequence of elementary moves relating the two, such that all the corresponding maps $\sigma_{i}^{ \pm 1}$ preserve the desired properties. This will reduce our statements to the bipartite case. Our results will then follow from another important property of the set of $c$-almost-positive roots: when the Coxeter element is bipartite, there exists a bijection

$$
t_{-}: \Phi_{\geq-1} \rightarrow \Phi_{\text {ap }}(t)
$$

which is induced by a linear map. This will allow us, in this particular case, to deduce our results from their analogs from [6] and [18]. 
As a byproduct of the construction we get an explicit description of the exchange relations of $\mathcal{A}_{0}(c)$. Two cluster variables $x_{\lambda, c}$ and $x_{\mu, c}$ in it are exchangeable if and only if $(\lambda \| \mu)_{c}^{\Pi}=1=(\mu \| \lambda)_{c}^{\Pi}$. Denote by $T$ the cyclic group generated by $\tau_{c}^{\Pi}$. The proof of Theorem 4 relies on the fact that, except in some degenerate cases, for any pair of weights $\lambda$ and $\mu$ in $\Pi(c)$ corresponding to a pair of exchangeable cluster variables, the set

$$
\left\{\tau\left(\tau^{-1}(\lambda)+\tau^{-1}(\mu)\right)\right\}_{\tau \in T}
$$

consists of two vectors: $\lambda+\mu$ and another one denoted by $\lambda \uplus_{c} \mu$.

Use Theorem 3 to label all cluster monomials in $\mathcal{A}_{0}(c)$ by points of $P$ :

$$
x_{\sum m_{\lambda} \lambda, c}:=\prod x_{\lambda, c}^{m_{\lambda}} .
$$

Theorem 5 All the exchange relations in $\mathcal{A}_{0}(c)$ are of the form

$$
x_{\lambda, c} x_{\mu, c}=x_{\lambda+\mu, c}+x_{\lambda \uplus_{c} \mu, c} .
$$

We now discuss the connection of $\mathcal{F}_{c}^{\Pi}$ with the Cambrian fan defined in [14]. First recall some definitions and results from [11].

Let $D$ be the fundamental Weyl chamber, i.e., the $\mathbb{R}_{+}$-span of the fundamental weights. The Coxeter fan $\mathcal{F}$ is the complete simplicial fan in $P_{\mathbb{R}}$ whose maximal cones are the images of $D$ under the action of $W$. It is well known that the correspondence

$$
w \mapsto w(D)
$$

is a bijection between $W$ and the set of maximal cones of $\mathcal{F}$; moreover $\mathcal{F}$ is the normal fan to a distinguished polytope: the permutahedron (see e.g. [12]).

Using the (right) weak order, $W$ can be regarded as a lattice with minimal and maximal element $e$ and $w_{0}$, respectively. To each lattice congruence on $W$ corresponds a fan that coarsens $\mathcal{F}$ as shown in [13]; maximal cones in the new fan are obtained gluing together cones of $\mathcal{F}$ corresponding to elements of $W$ belonging to the same equivalence class.

Fix a Coxeter element $c$ and one of its reduced expressions. For any subset $J \subset I$, denote by $c_{J}$ the sub-word of $c$ obtained omitting the simple reflections $\left\{s_{i}\right\}_{i \in I \backslash J}$. Let $c^{\infty}$ be the formal word obtained concatenating infinitely many copies of $c$. Every reduced expression of $w \in W$ can be seen as a sub-word of $c^{\infty}$; call the $c$-sorting word of $w$ the lexicographically first sub-word of $c^{\infty}$ realizing it. The $c$-sorting word of $w$ can be encoded by a sequence of subsets $I_{1}, I_{2}, \ldots I_{k}$ of $I$ (the $c$-factorization of $w$ ) so that

$$
w=c_{I_{1}} c_{I_{2}} \cdots c_{I_{k}} \text {. }
$$

Note that the $c$-factorization of $w$ is independent on the reduced expression chosen for $c$ : it depends only on the Coxeter element itself. 
Definition 1 An element $w$ in $W$ is

- $c$-sortable if its $c$-factorization is such that

$$
I_{1} \supseteq I_{2} \supseteq \cdots \supseteq I_{k}
$$

- c-antisortable if $w w_{0}$ is $c^{-1}$-sortable

As an example pick $c=s_{1} s_{2} s_{3}$ in type $A_{3}$, then $s_{2} s_{3} s_{2}$ is $c$-sortable with the $c$ factorization $\{2,3\},\{2\}$, the element $s_{2} s_{3} s_{1} s_{2} s_{1}$ is $c$-antisortable while $s_{2} s_{3} s_{2} s_{1}$ is neither.

For any element $w$ in $W$, again in the weak order, there exist a unique minimal $c$ antisortable element above it and a unique maximal $c$-sortable below it; denote them by $\pi_{c}^{\uparrow}(w)$ and $\pi_{\downarrow}^{c}(w)$, respectively.

Proposition 2 (cf. [15]) For any $w \in W$ the sets

$$
\left(\pi_{\downarrow}^{c}\right)^{-1}\left(\pi_{\downarrow}^{c}(w)\right)
$$

and

$$
\left(\pi_{c}^{\uparrow}\right)^{-1}\left(\pi_{c}^{\uparrow}(w)\right)
$$

coincide; they are intervals in the lattice $W$ with minimal element $\pi_{\downarrow}^{c}(w)$ and maximal element $\pi_{c}^{\uparrow}(w)$.

Define a lattice congruence on $W$ by setting

$$
v \sim w \quad \Leftrightarrow \quad \pi_{\downarrow}^{c}(v)=\pi_{\downarrow}^{c}(w) .
$$

The $c$-Cambrian fan $\mathcal{F}_{c}^{C}$ (defined in [16]) is the complete simplicial fan obtained from $\mathcal{F}$ by coarsening with respect to the lattice congruence (5); its maximal cones are parametrized by $c$-sortable elements.

In [11] it was shown that, for any point $a$ in the fundamental Weyl chamber, there is a unique simple polytope $\operatorname{Asso}_{c}^{a}(W)$ with normal fan $\mathcal{F}_{c}^{C}$ and such that $a$ is a vertex of $\operatorname{Asso}_{c}^{a}(W)$.

We have now all the required notations to state our last result.

Theorem 6 For every $f: I \rightarrow \mathbb{R}$ satisfying the hypothesis of Theorem 4 there exists a point $a \in D$ such that the polytopes $\operatorname{Asso}_{c}^{a}(W)$ and $\operatorname{Asso}_{c}^{f}(W)$ coincide.

As a direct consequence we get

Corollary 1 The c-Cambrian fan $\mathcal{F}_{c}^{C}$ and the c-cluster fan $\mathcal{F}_{c}^{\Pi}$ coincide. 


\section{The set $\Phi_{\text {ap }}(c)$}

Fix a Dynkin diagram $I$ and let $\Phi=\Phi_{+} \sqcup \Phi_{-}$be the corresponding root system. For convenience we identify $I$ with $\{1, \ldots, n\}$ so that a chosen Coxeter element is $c=s_{1} \cdots s_{n}$.

For $i \in I$ let

$$
\beta_{i}^{c}:=s_{n} \cdots s_{i+1} \alpha_{i}
$$

Remark 5 It is known that the roots (6) are exactly the positive roots that are mapped into negative roots by $c$; moreover they form a $\mathbb{Z}$-basis of the root lattice $Q$ since the linear map sending each $\alpha_{i}$ to $\beta_{i}^{c}$ is unitriangular.

We call $\Phi_{\text {ap }}(c):=\Phi_{+} \cup\left\{-\beta_{i}^{c}\right\}_{i \in I}$ the set of the $c$-almost-positive roots and define a bijection $\tau_{c}^{\Phi}: \Phi_{\text {ap }}(c) \rightarrow \Phi_{\text {ap }}(c)$ by setting, for $\alpha \in \Phi_{\text {ap }}(c)$,

$$
\tau_{c}^{\Phi}(\alpha):= \begin{cases}-\beta_{i}^{c} & \text { if } \alpha=\beta_{i}^{c} \\ c \alpha & \text { otherwise }\end{cases}
$$

Definition 2 The $c$-compatibility degree on $\Phi_{\text {ap }}(c)$ is the unique $\tau_{c}^{\Phi}$-invariant function

$$
(\bullet \mid \bullet)_{c}^{\Phi}: \Phi_{\mathrm{ap}}(c) \times \Phi_{\mathrm{ap}}(c) \longrightarrow \mathbb{N}
$$

defined by the initial conditions

$$
\left(-\beta_{i}^{c} \| \alpha\right)_{c}^{\Phi}:=\left[\alpha ; \alpha_{i}\right]_{+}
$$

These definitions are justified by the following proposition.

Proposition 3 The linear map

$$
\phi_{c}:=\left(c^{-1}-1\right): P_{\mathbb{R}} \longrightarrow Q_{\mathbb{R}}
$$

is invertible and restricts to an isomorphism of the weight lattice $P$ with the root lattice $Q$ sending $\Pi(c)$ to $\Phi_{\mathrm{ap}}(c)$. Moreover $\phi_{c}$ intertwines $\tau_{c}^{\Pi}$ and $\tau_{c}^{\Phi}$ and transform the compatibility degree $(\bullet \| \bullet)_{c}^{\Pi}$ on $\Pi(c)$ into the compatibility degree $(\bullet \| \bullet)_{c}^{\Phi}$ on $\Phi_{\text {ap }}(c)$.

Proof To show that $\phi_{c}$ is a lattice isomorphism, in view of Remark 5, it suffices to establish that

$$
\phi_{c}\left(\omega_{i}\right)=-\beta_{i}^{c}
$$

Using the well-known property

$$
s_{i} \omega_{j}= \begin{cases}\omega_{i}-\alpha_{i} & \text { if } i=j \\ \omega_{j} & \text { otherwise }\end{cases}
$$


we have

$$
\phi_{c}\left(\omega_{i}\right)=s_{n} \cdots s_{1} \omega_{i}-\omega_{i}=s_{n} \cdots s_{i+1}\left(s_{i} \omega_{i}-\omega_{i}\right)=s_{n} \cdots s_{i+1}\left(-\alpha_{i}\right)=-\beta_{i}^{c} .
$$

The sets $\Pi(c)$ and $\Phi_{\text {ap }}(c)$ have the same cardinality. Indeed Proposition 1.7 in [18] states that, for every $i$, the sum $h(i, c)+h\left(i^{*}, c\right)$ is equal to the Coxeter number $h$, hence

$$
|\Pi(c)|=\sum_{i \in I}(h(i, c)+1)=\frac{1}{2} \sum_{i \in I}\left(h(i, c)+h\left(i^{*}, c\right)+2\right)=\frac{1}{2} \sum_{i \in I}(h+2)=\left|\Phi_{\mathrm{ap}}(c)\right| .
$$

To conclude the proof of the first part it suffices to check that any weight in $\Pi(c) \backslash$ $\left\{\omega_{i}\right\}_{i \in I}$ is mapped to a positive root. This was already showed in [18] during the proof of the inequalities (1.8) in it.

To show that, for any $\alpha \in \Phi_{\text {ap }}(c)$,

$$
\phi_{c}^{-1}\left(\tau_{c}^{\Phi}(\alpha)\right)=\tau_{c}^{\Pi}\left(\phi_{c}^{-1}(\alpha)\right)
$$

there are two cases to consider:

1. if $\alpha=\beta_{i}^{c}$ then

$$
\phi_{c}^{-1}\left(\tau_{c}^{\Phi}\left(\beta_{i}^{c}\right)\right)=\phi_{c}^{-1}\left(-\beta_{i}^{c}\right)=\omega_{i}=\tau_{c}^{\Pi}\left(-\omega_{i}\right)=\tau_{c}^{\Pi}\left(\phi_{c}^{-1}\left(\beta_{i}^{c}\right)\right)
$$

2. if $\alpha \neq \beta_{i}^{c}$ for any $i$ then

$$
\phi_{c}^{-1}\left(\tau_{c}^{\Phi}(\alpha)\right)=\phi_{c}^{-1}(c \alpha)=\left(c^{-1}-1\right)^{-1} c \alpha=c\left(c^{-1}-1\right)^{-1} \alpha=\tau_{c}^{\Pi}\left(\phi_{c}^{-1}(\alpha)\right) .
$$

To conclude the proof it is sufficient to show that both compatibility degrees satisfy the same initial conditions. On the one hand we have

$$
\left(-\beta_{i}^{c} \| \alpha\right)_{c}^{\Phi}=\left[\alpha ; \alpha_{i}\right]_{+}
$$

and on the other

$$
\left(\phi_{c}^{-1}\left(-\beta_{i}^{c}\right) \| \phi_{c}^{-1}(\alpha)\right)_{c}^{\Pi}=\left(\omega_{i} \| \phi_{c}^{-1}(\alpha)\right)_{c}^{\Pi}=\left[\left(c^{-1}-1\right)\left(c^{-1}-1\right)^{-1} \alpha ; \alpha_{i}\right]_{+} .
$$

Remark 6 As in the case of $\Pi(c)$ the action of $\tau_{c}^{\Phi}$ on $\Phi_{\text {ap }}(c)$ and the action of $w_{0}$ on $I$ are compatible, i.e. there exists $m \in \mathbb{Z}$ such that

$$
\left(\tau_{c}^{\Phi}\right)^{m}\left(-\beta_{i}^{c}\right)=-\beta_{j}^{c}
$$

if and only if $j=i$ or $j=i^{*}$.

We can now rephrase Theorems 2, 3, 4, 5, and Proposition 1 in this new setup.

Let $\Delta_{c}^{\Phi}$ be the abstract simplicial complex having elements of $\Phi_{\text {ap }}(c)$ as vertices and with subsets of pairwise compatible roots as simplices; similarly to the case of $\Pi(c)$, we call $c$-clusters the maximal (by inclusion) simplices.

In view of Proposition 3, Theorem 2 is equivalent to the following. 
Theorem 7 Each c-cluster in $\Delta_{c}^{\Phi}$ is a $\mathbb{Z}$-basis of the root lattice $Q$.

Definition 3 For any $\gamma$ in $Q$ we call a $c$-cluster expansion of $\gamma$ an expression

$$
\gamma=\sum_{\alpha \in \Phi_{\mathrm{ap}}(c)} m_{\alpha} \alpha
$$

where all the coefficients $m_{\alpha}$ are non-negative integers such that $m_{\alpha} m_{\delta}=0$ whenever $(\alpha \| \delta)_{c}^{\Phi} \neq 0$.

The counterpart of Proposition 1 is the following:

Proposition 4 Any $\gamma$ in the root lattice $Q$ admits a unique c-cluster expansion.

Remark 7 Our proof of Proposition 4 will mimic, step by step, the proof of Theorem 3.11 in [3]. A sketch of a different proof, more similar to the others in this paper, will be also given.

Let $\mathcal{F}_{c}^{\Phi}$ be the set of all the cones in the space $Q_{\mathbb{R}}$ that are the positive linear span of simplices of the complex $\Delta_{c}^{\Phi}$. A direct consequence of Proposition 4 is the following counterpart of Theorem 3 .

Theorem $8 \mathcal{F}_{c}^{\Phi}$ is a complete simplicial fan.

As for the case of $\Pi(c)$, once Theorem 8 is established, any function defined on $\Phi_{\text {ap }}(c)$ can be extended to a continuous, piecewise-linear function on $Q_{\mathbb{R}}$ that is linear on the maximal cones of $\mathcal{F}_{c}^{\Phi}$. In particular, any function

$$
f: I \longrightarrow \mathbb{R}
$$

such that $f(i)=f\left(i^{*}\right)$ gives rise to a $\tau_{c}^{\Phi}$-invariant, continuous, piecewise-linear function

$$
F_{c}=F_{c ; f}: Q_{\mathbb{R}} \longrightarrow \mathbb{R}
$$

by setting

$$
F_{c}\left(-\beta_{i}^{c}\right):=f(i)
$$

and extending, first to $\Phi_{\text {ap }}(c)$ and then to $Q_{\mathbb{R}}$, as prescribed.

Let $\operatorname{Asso}_{c}^{f, \Phi}(W)$ be the subset of $Q_{\mathbb{R}}^{*}$ defined by

$$
\operatorname{Asso}_{c}^{f, \Phi}(W):=\left\{\varphi \in Q_{\mathbb{R}}^{*} \mid \varphi(\alpha) \leq F_{c}(\alpha) \forall \alpha \in \Phi_{\text {ap }}(c)\right\} .
$$

Theorem 9 If $f: I \rightarrow \mathbb{R}$ is such that

1. for any $i \in I$

$$
f(i)=f\left(i^{*}\right)
$$


2. for any $j \in J$

$$
\sum_{i \in I} a_{i j} f(i)>0
$$

then $\operatorname{Asso}_{c}^{f, \Phi}(W)$ is a simple n-dimensional polytope with support function $F_{c}$. Furthermore, the domains of linearity of $F_{c}$ are exactly the maximal cones of $\mathcal{F}_{c}^{\Phi}$, hence the normal fan of $\operatorname{Asso}_{c}^{f, \Phi}(W)$ is $\mathcal{F}_{c}^{\Phi}$.

Again by Proposition 3, Theorem 9 implies Theorem 4.

The proof of Theorem 9 is based on an explicit characterization of the roots in $\Phi_{\text {ap }}(c)$ belonging to adjacent maximal cones of $\mathcal{F}_{c}^{\Phi}$. Namely there exist two $c$ clusters $C_{\alpha}$ and $C_{\gamma}$ such that $C_{\alpha} \backslash\{\alpha\}=C_{\gamma} \backslash\{\gamma\}$ if and only if

$$
(\alpha \| \gamma)_{c}^{\Phi}=1=(\gamma \| \alpha)_{c}^{\Phi}
$$

(cf. Lemma 9). For all such pairs of roots the set

$$
\left\{\left(\tau_{c}^{\Phi}\right)^{-m}\left(\left(\tau_{c}^{\Phi}\right)^{m}(\alpha)+\left(\tau_{c}^{\Phi}\right)^{m}(\beta)\right)\right\}_{m \in \mathbb{Z}}
$$

consists (when $I$ has no connected component with only one node) of precisely two vectors, $\alpha+\gamma$ and $\alpha \uplus_{c} \gamma$; their $c$-cluster expansion are supported on $C_{\alpha} \cap C_{\gamma}$ and they are disjoint (cf. Proposition 14 and Corollary 4).

Let $\mathcal{A}_{0}(c)$ the coefficient-free cluster algebra with initial orientation given by $c$; label its cluster variables by roots in $\Phi_{\text {ap }}(c)$ and, in view of Proposition 4 , its cluster monomials by points in the root lattice. Using this notation Theorem 5 can be restated as follows.

Theorem 10 All the exchange relations in $\mathcal{A}_{0}(c)$ are of the form

$$
x_{\alpha, c} x_{\gamma, c}=x_{\alpha+\gamma, c}+x_{\alpha \uplus_{c} \gamma, c}
$$

for suitable c-almost positive roots $\alpha$ and $\gamma$ such that

$$
(\alpha \| \gamma)_{c}^{\Phi}=1=(\gamma \| \alpha)_{c}^{\Phi}
$$

As mentioned before the main advantage of the labels $\Phi_{\mathrm{ap}}(c)$ over $\Pi(c)$ is that it is easier to set up inductions on $|I|$. Let $J \subset I$ be a sub-diagram of $I$. Fix a Coxeter element $c$ for $I$ and denote by $c_{J}$ the sub-word of $c$ obtained omitting all the simple reflections $\left\{s_{i}\right\}_{i \in I \backslash J}$. By construction $c_{J}$ is a Coxeter element in the Weyl group $W_{J}$ (we denote by $W_{J}$ the standard parabolic subgroup of $W$ generated by $\left\{s_{j}\right\}_{j \in J}$ ). Let

$$
\iota=\iota_{c}: \Phi_{\mathrm{ap}}^{J}\left(c_{J}\right) \longrightarrow \Phi_{\mathrm{ap}}(c)
$$

be the "twisted" inclusion map given by

$$
\iota(\alpha):= \begin{cases}-\beta_{i}^{c} & \text { if } \alpha=-\beta_{i}^{c_{J}}, i \in J \\ \alpha & \text { otherwise. }\end{cases}
$$


From this moment on, unless it is not clear from the context, superscripts $\Phi$ and $\Pi$ will be omitted in order to make notation less heavy. this:

Denote by $(\bullet \| \bullet)_{c_{J}}^{J}$ the $c_{J}$-compatibility degree on $\Phi_{\text {ap }}^{J}\left(c_{J}\right)$. The key property is

Proposition 5 Let $\alpha$ and $\gamma$ be roots in $\Phi_{\mathrm{ap}}^{J}\left(c_{J}\right)$. Then

$$
(\iota(\alpha) \| \iota(\gamma))_{c}=(\alpha \| \gamma)_{c_{J}}^{J}
$$

Remark 8 In the setup of almost positive roots the analog of this statement is point 3 of Proposition 3.3 in [6]; there the map $\iota$ is the ordinary inclusion. A proof of Proposition 5 will be given in Sect. 5 .

The original construction in [6] does not distinguish among the possible bipartite orientations of $I$. With this motivation in mind consider the map $\alpha \mapsto \bar{\alpha}$ between $\Phi_{\text {ap }}(c)$ and $\Phi_{\text {ap }}\left(c^{-1}\right)$ defined by

$$
\bar{\alpha}:= \begin{cases}-\beta_{i}^{c^{-1}} & \text { if } \alpha=-\beta_{i}^{c}, i \in I \\ \alpha & \text { otherwise. }\end{cases}
$$

Proposition 6 For any $\alpha$ and $\gamma$ in $\Phi_{\mathrm{ap}}(c)$

$$
(\alpha \| \gamma)_{c}=(\bar{\alpha} \| \bar{\gamma})_{c^{-1}}
$$

Proof Initial conditions agree:

$$
\left(-\beta_{i}^{c} \| \alpha\right)_{c}=\left[\alpha ; \alpha_{i}\right]_{+}=\left[\bar{\alpha} ; \alpha_{i}\right]_{+}=\left(-\beta_{i}^{c^{-1}} \| \bar{\alpha}\right)_{c^{-1}}=\left(\overline{-\beta_{i}^{c}} \| \bar{\alpha}\right)_{c^{-1}} .
$$

It suffices then to show that, for any $\alpha \in \Phi_{\mathrm{ap}}(c)$,

$$
\overline{\tau_{c}(\alpha)}=\tau_{c^{-1}}^{-1}(\bar{\alpha})
$$

There are three cases to be considered.

1. If $\alpha=-\beta_{i}^{c}$ for some $i \in I$ then on the one hand

$$
\overline{\tau_{c}\left(-\beta_{i}^{c}\right)}=\overline{-c \beta_{i}^{c}}=\overline{-s_{1} \cdots s_{n}\left(s_{n} \cdots s_{i+1} \alpha_{i}\right)}=\overline{s_{1} \cdots s_{i-1} \alpha_{i}}=\beta_{i}^{c^{-1}} ;
$$

on the other hand

$$
\tau_{c^{-1}}^{-1}\left(\overline{-\beta_{i}^{c}}\right)=\tau_{c^{-1}}^{-1}\left(-\beta_{i}^{c^{-1}}\right)=\beta_{i}^{c^{-1}}
$$

2. When $\alpha=\beta_{i}^{c}$

$$
\overline{\tau_{c}\left(\beta_{i}^{c}\right)}=\overline{-\beta_{i}^{c}}=-\beta_{i}^{c^{-1}}=s_{1} \cdots s_{i} \alpha_{i}
$$

multiplying and dividing by $s_{i+1} \cdots s_{n}$ we get

$$
s_{1} \cdots s_{i}\left(s_{i+1} \cdots s_{n} s_{n} \cdots s_{i+1}\right) \alpha_{i}=\left(c^{-1}\right)^{-1} \beta_{i}^{c}=\tau_{c^{-1}}^{-1}\left(\beta_{i}^{c}\right)=\tau_{c^{-1}}^{-1}\left(\overline{\beta_{i}^{c}}\right) .
$$


3. Finally for $\alpha \neq \pm \beta_{i}^{c}$

$$
\overline{\tau_{c}(\alpha)}=\overline{c \alpha}=c \alpha=\left(c^{-1}\right)^{-1} \alpha=\tau_{c^{-1}}^{-1}(\bar{\alpha}) .
$$

\section{The bipartite case}

In this section we assume that the Dynkin diagram $I$ is connected; the statements in the general case are easily reduced to this. Since any connected Dynkin diagram is a tree, we can split $I$ into two disjoint subsets $I_{+}$and $I_{-}$such that every edge in it has one endpoint in $I_{+}$and one in $I_{-}$. Up to relabeling, this can be done in a unique way. A bipartite Coxeter can thus be written as

$$
t=t_{\varepsilon} t_{-\varepsilon}
$$

where $\varepsilon$ denotes a sign and

$$
t_{\varepsilon}:=\prod_{i \in I_{\varepsilon}} s_{i}
$$

(the expression makes sense since the factors commute with each other). By our assumption there are precisely two bipartite Coxeter elements in $W: t=t_{+} t_{-}$and $t^{-1}=t_{-} t_{+}$.

Let $\Phi_{\geq-1}$ be the set of almost positive roots, i.e.

$$
\Phi_{\geq-1}:=\Phi_{+} \cup\left\{-\alpha_{i}\right\}_{i \in I}
$$

introduced in [6] to parametrize cluster variables in the special case of a bipartite initial cluster. On it there are two involutions $\tau_{+}$and $\tau_{-}$defined by

$$
\tau_{\varepsilon}(\alpha)= \begin{cases}\alpha & \text { if } \alpha=-\alpha_{i} \text { and } i \in I_{-\varepsilon} \\ t_{\varepsilon} \alpha & \text { otherwise }\end{cases}
$$

and a unique $\left\{\tau_{+}, \tau_{-}\right\}$-invariant compatibility degree function $(\bullet \| \bullet)_{\geq-1}$ satisfying

$$
\left(-\alpha_{i}|| \gamma\right)_{\geq-1}=\left[\gamma ; \alpha_{i}\right]_{+}
$$

Call two almost positive roots $\alpha$ and $\gamma$ compatible if

$$
(\alpha \| \gamma)_{\geq-1}=0=(\gamma \| \alpha)_{\geq-1}
$$

The cluster complex $\Delta_{\geq-1}$ is the abstract simplicial complex induced on $\Phi_{\geq-1}$ by the compatibility degree function; its simplices are subsets of pairwise compatible almost-positive roots. As before call the maximal simplices clusters and consider the set $\mathcal{F}_{\geq-1}$ of all simplicial cones generated by simplices.

As we mentioned in the introduction our construction is based on the results for the bipartite case given in [6] and [3]. From the first paper we will need the following. 


\section{Proposition 7}

1. [Proposition 3.3 (2)] For any pair of almost positive roots $\alpha$ and $\gamma$, we have

$$
(\alpha|| \gamma)_{\geq-1}=0
$$

if and only if $(\gamma \| \alpha)_{\geq-1}=0$.

2. [Proposition $3.3(3)$ ] Let $J$ be a subset of I and denote by $\Phi_{\geq-1}^{J}$ the corresponding set of almost positive roots. Let $\alpha$ and $\gamma$ be roots in $\Phi_{\geq-1}^{J}$, then

$$
(\alpha \| \gamma)_{\geq-1}=(\alpha \| \gamma)_{\geq-1}^{J}
$$

where $(\bullet \| \bullet)_{\geq-1}^{J}$ denotes the compatibility degree function on $\Phi_{\geq-1}^{J}$.

3. [Theorem 1.8] Each cluster in the cluster complex is a $\mathbb{Z}$-basis of the root lattice $Q$.

4. [Theorem 3.11] Any $\gamma \in Q$ admits a unique cluster expansion. In other words $\gamma$ can uniquely be written as

$$
\gamma=\sum_{\alpha \in \Phi_{\geq-1}} m_{\alpha} \alpha
$$

so that all the coefficients $m_{\alpha}$ are non-negative integers and $m_{\alpha} m_{\alpha^{\prime}}=0$ if $\left(\alpha|| \alpha^{\prime}\right)_{>-1} \neq 0$.

5. [Theorem 1.10] $\mathcal{F}_{\geq-1}$ is a complete simplicial fan in $Q_{\mathbb{R}}$.

The results we will need from [3] can be summarized as follows.

Proposition 8 Suppose that I has at least two vertices. Let $\alpha$ and $\gamma$ be almost positive roots such that $(\alpha \| \gamma)_{\geq-1}=1=(\gamma|| \alpha)_{\geq-1}$. Then we have:

1. [Theorem 1.14] The set

$$
\left\{\tau\left(\tau^{-1}(\alpha)+\tau^{-1}(\gamma)\right)\right\}_{\tau \in T}
$$

where $T$ denotes the group generated by $\tau_{+}$and $\tau_{-}$, consists of exactly two elements $\alpha+\gamma$ and $\alpha \uplus \gamma$.

2. [Lemma 2.3] Any root appearing with a positive coefficient in the cluster expansion of $\alpha+\gamma$ or $\alpha \uplus \gamma$ is compatible with both $\alpha, \gamma$, and with any other root compatible with both $\alpha$ and $\gamma$.

3. [Lemma 2.4] Let $f: I \rightarrow \mathbb{R}_{+}$be any function such that, for any $i \in I$,

$$
f(i)=f\left(i^{*}\right)
$$

and

$$
\sum_{i \in I} a_{i j} f(i)>0
$$

for any $j \in I$. Let $F_{\geq-1}: Q_{\mathbb{R}} \rightarrow \mathbb{R}$ be the continuous piecewise-linear function on $Q_{\mathbb{R}}$ that is linear on the maximal cones of $\mathcal{F}_{\geq-1}$, invariant under the action of $T$, 
and such that

$$
F_{\geq-1}\left(-\alpha_{i}\right)=f(i) \text {. }
$$

Then,

$$
F_{\geq-1}(\alpha)+F_{\geq-1}(\gamma)>\max \left\{F_{\geq-1}(\alpha+\gamma), F_{\geq-1}(\alpha \uplus \gamma)\right\}
$$

The statements regarding $\alpha \uplus \gamma$ are not expressed explicitly in [3] but can be recovered immediately from the corresponding statements about $\alpha+\gamma$. Indeed, let $\tau$ be such that

$$
\alpha \uplus \gamma=\tau^{-1}(\tau(\alpha)+\tau(\gamma)) .
$$

Any root appearing with positive coefficient in the cluster expansion of $\tau(\alpha \uplus \gamma)$ is compatible with $\tau(\alpha), \tau(\gamma)$ and with any root compatible with both. Since $\tau$ preserves the compatibility degree we get 2 . Similarly for 3 :

$$
F_{\geq-1}(\tau(\alpha))+F_{\geq-1}(\tau(\gamma))>F_{\geq-1}(\tau(\alpha)+\tau(\gamma))=F_{\geq-1}(\tau(\alpha \uplus \gamma))
$$

since $F_{\geq-1}$ is invariant under the action of $\tau$ we can conclude

$$
F_{\geq-1}(\alpha)+F_{\geq-1}(\gamma)>F_{\geq-1}(\alpha \uplus \gamma) .
$$

We need to translate the above results to $\Phi_{\text {ap }}(t)$; in order to do so we need a bijection between $\Phi_{\geq-1}$ and $\Phi_{\text {ap }}(t)$ induced by a linear map. Note that Proposition 3 together with Lemma 5.2 in [18] already provide a bijection but it is not induced by a linear map.

Note also that, for a bipartite Coxeter element $t=t_{+} t_{-}$, the negative roots in $\Phi_{\text {ap }}(t)$ are the roots $-\beta_{i}^{t}$ given by

$$
-\beta_{i}^{t}= \begin{cases}-\alpha_{i} & i \in I_{-} \\ -t_{-} \alpha_{i} & i \in I_{+}\end{cases}
$$

Proposition 9 The linear involution

$$
t_{-}: Q_{\mathbb{R}} \longrightarrow Q_{\mathbb{R}}
$$

restricts to an automorphism of $Q$ and to a bijection

$$
t_{-}: \Phi_{\geq-1} \longrightarrow \Phi_{\mathrm{ap}}(t) .
$$

Proof It suffices to show that, for any root $\alpha$ in $\Phi_{\geq-1}$, we have $t_{-}(\alpha) \in \Phi_{\text {ap }}(t)$. Let us first deal with roots whose image is negative.

- If $i \in I_{+}$, then

$$
t_{-}\left(-\alpha_{i}\right)=-t_{-} \alpha_{i}=-\beta_{i}^{t} .
$$

- If $i \in I_{-}$, then

$$
t_{-}\left(\alpha_{i}\right)=s_{i} \alpha_{i}=-\alpha_{i}=-\beta_{i}^{t} .
$$


This also shows that $t_{-}\left(-\alpha_{i}\right)=\beta_{i}^{t}$ if $i \in I_{-}$. For any other root $\alpha$ in $\Phi_{\geq-1}$, that is, for any positive root not in $\left\{\alpha_{i}\right\}_{i \in I_{-}}$, the image $t_{-}(\alpha)$ is positive since the support of any positive root is a connected sub-diagram of the Dynkin diagram and $t_{-}$sends any root to itself plus a linear combination of simple roots indexed by $I_{-}$.

Proposition 10 The map $t_{-}$intertwines $\tau_{t}$ with $\tau_{-} \tau_{+}$and preserves compatibility degree. In other words, for any almost-positive roots $\alpha$ and $\gamma$, we have

$$
\tau_{t}\left(t_{-} \alpha\right)=t_{-} \tau_{-} \tau_{+}(\alpha)
$$

and

$$
(\alpha|| \gamma)_{\geq-1}=\left(t_{-} \alpha \| t_{-} \gamma\right)_{t}
$$

Proof Proceed by direct inspection;

- if $t_{-} \alpha=\alpha_{i}=\beta_{i}^{t}$ for $i \in I_{-}$, that is if $\alpha=-\alpha_{i}$ with $i \in I_{-}$, then

$$
\tau_{t}\left(t_{-}\left(-\alpha_{i}\right)\right)=\tau_{t}\left(\beta_{i}^{t}\right)=-\beta_{i}^{t}=-\alpha_{i}=t_{-} \alpha_{i}=t_{-} \tau_{-} \tau_{+}\left(-\alpha_{i}\right)
$$

- if $t_{-} \alpha=\beta_{i}^{t}$ for $i \in I_{+}$, i.e. if $\alpha=\alpha_{i}$ with $i \in I_{+}$, then

$$
\tau_{t}\left(t_{-} \alpha_{i}\right)=\tau_{t}\left(\beta_{i}^{t}\right)=-\beta_{i}^{t}=-t_{-} \alpha_{i}=t_{-}\left(-\alpha_{i}\right)=t_{-} \tau_{-} \tau_{+}\left(\alpha_{i}\right)
$$

- in any other case

$$
\tau_{t}\left(t_{-} \alpha\right)=t t_{-} \alpha=t_{+} \alpha=t_{-} t_{-} t_{+} \alpha=t_{-} \tau_{-} \tau_{+}(\alpha) .
$$

To conclude the proof it is enough to show that

$$
\left(-\alpha_{i} \| \gamma\right)_{\geq-1}=\left(-t_{-} \alpha_{i} \| t_{-} \gamma\right)_{t}
$$

for any $\gamma \in \Phi_{\geq-1}$ and any $i \in I$. If $i$ is in $I_{+}$then

$$
\left(-\alpha_{i} \| \gamma\right)_{\geq-1}=\left[\gamma ; \alpha_{i}\right]_{+}=\left[t_{-} \gamma ; \alpha_{i}\right]_{+}=\left(-\beta_{i}^{t} \| t_{-} \gamma\right)_{t}=\left(t_{-}\left(-\alpha_{i}\right) \| t_{-} \gamma\right)_{t}
$$

where the second equality holds because $t_{-}$does not contain $s_{i}$. If $i \in I_{-}$then, on the one hand we have $\left(-\alpha_{i} \| \gamma\right)_{\geq-1}=\left[\gamma ; \alpha_{i}\right]_{+}$on the other

$$
\left(t_{-}\left(-\alpha_{i}\right) \| t_{-} \gamma\right)_{t}=\left(\alpha_{i} \| t_{-} \gamma\right)_{t}=\left(\tau_{t} \alpha_{i} \| \tau_{t} t_{-} \gamma\right)_{t}=\left(-\beta_{i}^{t} \| \tau_{t} t_{-} \gamma\right)_{t}=\left[\tau_{t} t_{-} \gamma ; \alpha_{i}\right]_{+} .
$$

Now there are three cases:

1. if $\gamma$ is $\alpha_{j}$ with $j \in I_{+}$then $\tau_{t} t_{-} \gamma=\tau_{t}\left(\beta_{j}^{t}\right)=-\beta_{j}^{t}$ and

$$
\left[\alpha_{j} ; \alpha_{i}\right]_{+}=0=\left[-\beta_{j}^{t} ; \alpha_{i}\right]_{+} .
$$

2. If $\gamma$ is $-\alpha_{j}$ with $j \in I_{-}$then

$$
\tau_{t} t_{-} \gamma=\tau_{t}\left(\beta_{j}^{t}\right)=-\beta_{j}^{t}=-\alpha_{j}=\gamma .
$$


3. For any other $\gamma$ we have $\tau_{t} t_{-} \gamma=t t_{-} \gamma=t_{+} \gamma$ and

$$
\left[t_{+} \gamma ; \alpha_{i}\right]_{+}=\left[\gamma ; \alpha_{i}\right]_{+}
$$

since $s_{i}$ does not appear in $t_{+}$.

Since the map $t_{-}$is linear, all the properties of $\Phi_{\geq-1}$ translate to $\Phi_{\text {ap }}(t)$ :

\section{Corollary 2}

1. For any $\alpha$ and $\gamma$ in $\Phi_{\text {ap }}(t)$ we have $(\alpha \| \gamma)_{t}=0$ if and only if $(\gamma \| \alpha)_{t}=0$.

2. For any $J \subset I$ and any pair of roots $\alpha$ and $\gamma$ in $\Phi_{\text {ap }}^{J}\left(t_{J}\right)$

$$
(\iota \alpha \| \iota \gamma)_{t}=(\alpha \| \gamma)_{t_{J}}^{J}
$$

3. Each $t$-cluster in the simplicial complex $\Delta_{t}^{\Phi}$ is a $\mathbb{Z}$-basis of the root lattice $Q$.

4. Any $\gamma \in Q$ admits a unique $t$-cluster expansion. That is, $\gamma$ can be uniquely written as

$$
\gamma=\sum_{\alpha \in \Phi_{\mathrm{ap}}(t)} m_{\alpha} \alpha
$$

so that all the coefficients $m_{\alpha}$ are non-negative integers and $m_{\alpha} m_{\alpha^{\prime}}=0$ whenever $\left(\alpha \| \alpha^{\prime}\right)_{t} \neq 0$.

5. The set $\mathcal{F}_{t}$ is a complete simplicial fan in $Q_{\mathbb{R}}$.

Proof The only non trivial claim is (2); it is enough to show that it holds when $I \backslash J=$ $\{j\}$. Since $t$ is bipartite, $s_{j}$ is either initial or final (cf. (10)). Using Proposition 6 we can assume it is initial, i.e. $j \in I_{+}$. We have then

$$
(\iota \alpha \| \iota \gamma)_{t}=\left(t_{-} \iota \alpha \| t_{-} \iota \gamma\right)_{\geq-1}=\left(t_{-} \alpha \| t_{-} \gamma\right)_{\geq-1}^{J}=(\alpha \| \gamma)_{t_{J}}^{J}
$$

where the second equality holds since, for any root $\alpha$ in $\Phi_{\text {ap }}^{J}\left(t_{J}\right)$,

$$
\left[t_{-} \iota(\alpha) ; \alpha_{j}\right]=0
$$

hence $t_{-} \iota(\alpha)$ is in $\Phi^{J}$. Indeed if $\alpha$ is positive then $t_{-} \alpha$ contains $\alpha_{j}$ only if $\alpha$ does since $s_{j}$ does not appear in $t_{-}$; if $\alpha=-\beta_{i}^{t_{J}}$ with $i \in I_{-}$then $t_{-} \iota\left(-\beta_{i}^{t_{J}}\right)=\alpha_{i}$ and finally if $\alpha=-\beta_{i}^{t_{J}}$ with $i \in I_{+} \backslash\{j\}$ then $t_{-} \iota\left(-\beta_{i}^{t_{J}}\right)=-\alpha_{i}$.

Corollary 3 Suppose that I has at least 2 vertices. If $\alpha$ and $\gamma$ in $\Phi_{\mathrm{ap}}(t)$ are such that $(\alpha \| \gamma)_{t}=1=(\gamma \| \alpha)_{t}$, then

1. The set

$$
\left\{\tau_{t}^{m}\left(\tau_{t}^{-m}(\alpha)+\tau_{t}^{-m}(\gamma)\right)\right\}_{m \in \mathbb{Z}}
$$

consists of exactly two elements $\alpha+\gamma$ and $\alpha \uplus_{t} \gamma$. 
2. Any root appearing with a positive coefficient in the cluster expansion of $\alpha+\gamma$ or $\alpha \uplus_{t} \gamma$ is compatible with both $\alpha, \gamma$, and with any other root compatible with both $\alpha$ and $\gamma$.

3. Let $f: I \rightarrow \mathbb{R}_{+}$be any function such that, for any $i \in I$,

$$
f(i)=f\left(i^{*}\right)
$$

and

$$
\sum_{i \in I} a_{i j} f(i)>0
$$

for any $j \in I$. Let $F_{t}: Q_{\mathbb{R}} \rightarrow \mathbb{R}$ be the continuous piecewise-linear function on $Q_{\mathbb{R}}$ that is linear on the maximal cones of $\mathcal{F}_{t}$, invariant under the action of $\tau_{t}$, and such that

$$
F_{t}\left(-\beta_{i}^{t}\right)=f(i)
$$

Then,

$$
F_{t}(\alpha)+F_{t}(\gamma)>\max \left\{F_{t}(\alpha+\gamma), F_{t}(\alpha \uplus \gamma)\right\}
$$

\section{Some technical results}

As anticipated we need to lift elementary moves to the level of $\Phi_{\text {ap }}(c)$. We concentrate first on conjugation by initial simple reflections. Fix the Coxeter element $c=s_{1} \cdots s_{n}$ and consider the bijection

$$
\sigma_{1}: \Phi_{\mathrm{ap}}(c) \longrightarrow \Phi_{\mathrm{ap}}\left(s_{1} c s_{1}\right)
$$

defined by

$$
\sigma_{1}(\alpha):= \begin{cases}\alpha_{1}\left(=\beta_{1}^{s_{1} c s_{1}}\right) & \text { if } \alpha=-\beta_{1}^{c} \\ s_{1} \alpha & \text { otherwise. }\end{cases}
$$

Note that $\sigma_{1}$ sends $-\beta_{i}^{c}$ to $-\beta_{i}^{s_{1} c s_{1}}$ for any $i \neq 1$.

Proposition 11 The map $\sigma_{1}$ intertwines $\tau_{c}$ and $\tau_{s_{1} c s_{1}}$, i.e., for any $\alpha$ in $\Phi_{\mathrm{ap}}(c)$, we have

$$
\tau_{s_{1} c s_{1}}\left(\sigma_{1}(\alpha)\right)=\sigma_{1}\left(\tau_{c}(\alpha)\right) .
$$

Moreover it preserves the compatibility degree, i.e. for any $\alpha$ and $\gamma$ in $\Phi_{\mathrm{ap}}(c)$

$$
(\alpha \| \gamma)_{c}=\left(\sigma_{1} \alpha \| \sigma_{1} \gamma\right)_{s_{1} c s_{1}}
$$

Proof It suffices to notice that $\sigma_{1}$ is the composition

$$
\Phi_{\mathrm{ap}}(c) \stackrel{\phi_{c}^{-1}}{\longrightarrow} \Pi(c) \stackrel{\psi_{s_{1} c s_{1}, c}^{-1}}{\longrightarrow} \Pi\left(s_{1} c s_{1}\right) \stackrel{\phi_{s_{1} c s_{1}}}{\longrightarrow} \Phi_{\mathrm{ap}}\left(s_{1} c s_{1}\right)
$$


where $\psi_{s_{1} c s_{1}, c}^{-1}$ is the bijection

$$
\psi_{s_{1} c s_{1}, c}^{-1}(\lambda):= \begin{cases}-\omega_{1} & \text { if } \lambda=\omega_{1} \\ s_{1} \lambda & \text { otherwise }\end{cases}
$$

defined by Lemma 5.3 in [18] and $\phi_{c}$ is the map of Proposition 3. Indeed, if $\alpha \neq-\beta_{1}^{c}$ then

$$
\phi_{s_{1} c s_{1}} \circ \psi_{s_{1} c s_{1}, c}^{-1} \circ \phi_{c}^{-1}(\alpha)=\left(s_{1} c^{-1} s_{1}-1\right) s_{1}\left(c^{-1}-1\right)^{-1} \alpha=s_{1} \alpha=\sigma_{1}(\alpha)
$$

and

$$
\phi_{s_{1} c s_{1}} \circ \psi_{s_{1} c s_{1}, c}^{-1} \circ \phi_{c}^{-1}\left(-\beta_{1}^{c}\right)=\left(s_{1} c^{-1} s_{1}-1\right)\left(-\omega_{1}\right)=\alpha_{1}=\sigma_{1}\left(-\beta_{1}^{c}\right) .
$$
do.

The bijection $\sigma_{1}$ satisfies the desired property because all the maps that define it

To use simultaneously induction on the rank of $I$ and elementary moves we need to prove some type of compatibility between $\sigma_{1}$ and $\iota$. It suffices to inspect their interaction in the case when $\iota$ is induced removing only one node (say $i$ ) from $I$.

Proposition 12 For $J=I \backslash\{i\}$ and $i \neq 1$ let $c_{J}$ be the Coxeter element of $W_{J}$ obtained by deleting $s_{i}$ from $c$ and let $\sigma_{1}^{J}$ be the map corresponding to the conjugation by $s_{1}$ in $W_{J}$. For any root $\alpha$ in $\Phi_{\mathrm{ap}}^{J}\left(c_{J}\right)$ we have

$$
\sigma_{1}\left(\iota_{c}(\alpha)\right)=\iota_{s_{1} c s_{1}}\left(\sigma_{1}^{J}(\alpha)\right)
$$

Proof There are three cases to be considered.

1. If $\alpha=-\beta_{1}^{c_{J}}$ then

$$
\sigma_{1}\left(\iota_{c}\left(-\beta_{1}^{c_{J}}\right)\right)=\sigma_{1}\left(-\beta_{1}^{c}\right)=\alpha_{1}=\iota_{s_{1} c s_{1}}\left(\alpha_{1}\right)=\iota_{s_{1} c s_{1}}\left(\sigma_{1}^{J}\left(-\beta_{1}^{c_{J}}\right)\right) .
$$

2. If $\alpha=-\beta_{j}^{c_{J}}$ and $j \neq 1$ then $\iota_{c}\left(-\beta_{j}^{c_{J}}\right)=-\beta_{j}^{c}$ therefore

$$
\sigma_{1}\left(\iota_{c}\left(-\beta_{j}^{c_{J}}\right)\right)=-\beta_{j}^{s_{1} c s_{1}}=\iota_{s_{1} c s_{1}}\left(-\beta_{j}^{s_{1} c_{J} s_{1}}\right)=\iota_{s_{1} c s_{1}}\left(\sigma_{1}^{J}\left(-\beta_{j}^{c_{J}}\right)\right) .
$$

3. If $\alpha$ is positive

$$
\sigma_{1}\left(\iota_{c}(\alpha)\right)=\sigma_{1}(\alpha)=s_{1} \alpha=\iota_{s_{1} c s_{1}}\left(s_{1} \alpha\right)=\iota_{s_{1} c s_{1}}\left(\sigma_{1}^{J}(\alpha)\right) .
$$

The last equality holds since, $\alpha$ being positive, $s_{1} \alpha \neq \alpha_{1}$ and the third because if $s_{1} \alpha$ is not positive then $\alpha=\alpha_{1}$ and $-\beta_{1}^{s_{1} c s_{1}}=-\beta_{1}^{s_{1} c_{J} s_{1}}=-\alpha_{1}$.

Remark 9 The definition of $\sigma_{1}$ can be replicated to get the maps $\sigma_{i}$ corresponding to conjugation by any initial simple reflection $s_{i}$. It is clear that, to get the maps corresponding to elementary moves that conjugate $c$ by a final simple reflection, it suffices to consider the inverses $\sigma_{i}^{-1}$. 
As a first application of the elementary moves let us show that the definition of $c$-compatible pair of roots make sense.

Lemma 2 For any $\alpha$ and $\gamma$ in $\Phi_{\text {ap }}(c)$

$$
(\alpha \| \gamma)_{c}=0 \Leftrightarrow(\gamma \| \alpha)_{c}=0 .
$$

Proof If $c$ is bipartite the statement is true by point 1 in Corollary 2. It is then enough to show that the property is preserved under $\sigma_{i}^{ \pm 1}$. Suppose that it holds for $c=s_{1} \cdots s_{n}$. If $(\alpha \| \gamma)_{s_{1} c s_{1}}=0$ then

$$
\left(\sigma_{1}^{-1} \alpha \| \sigma_{1}^{-1} \gamma\right)_{c}=0=\left(\sigma_{1}^{-1} \gamma \| \sigma_{1}^{-1} \alpha\right)_{c} .
$$

Therefore $(\gamma \| \alpha)_{s_{1} c s_{1}}=0$.

Our next goal is to show that "distant" roots are compatible. We need to introduce some terminology. For any positive root $\alpha$ define its support to be the set

$$
\operatorname{Supp}(\alpha):=\left\{i \in I \mid\left[\alpha ; \alpha_{i}\right] \neq 0\right\}
$$

and extend the definition to $\Phi_{\text {ap }}(c)$ declaring

$$
\operatorname{Supp}\left(-\beta_{i}^{c}\right):=\{i\} .
$$

Remark 10 If $\alpha$ and $\gamma$ are roots with supports contained in two different connected components of $I$ then $(\alpha \| \gamma)_{c}=0$ since $\tau_{c}$ preserves connected components.

We can improve on Remark 10.

Definition 4 Call two roots $\alpha$ and $\gamma$ spaced if, for any $i \in \operatorname{Supp}(\alpha)$ and for any $j \in \operatorname{Supp}(\gamma), a_{i j}=0$.

Remark 11 Note that if $\left(-\beta_{i}^{c} \| \alpha\right)_{c} \neq 0$ then $\alpha$ and $-\beta_{i}^{c}$ are not spaced.

Proposition 13 Let $\alpha$ and $\gamma$ be roots in $\Phi_{\mathrm{ap}}(c)$. If $\alpha$ and $\gamma$ are spaced then

$$
(\alpha \| \gamma)_{c}=0
$$

Proof Using Remark 10 we can assume that $I$ is connected. If any of $\alpha$ and $\gamma$ is a negative root we are done by Lemma 2 and Remark 11. Let then both $\alpha$ and $\gamma$ be positive roots.

Supports of positive roots are connected subgraphs of the Dynkin diagram I. Since $\alpha$ and $\gamma$ are spaced, there must exist at least one vertex on the shortest path connecting $\operatorname{Supp}(\alpha)$ and $\operatorname{Supp}(\gamma)$ not belonging to either of the supports. Let $i$ be the nearest to $\operatorname{Supp}(\alpha)$ of such vertices. Let $I^{\prime}$ be a connected component of $I \backslash\{i\}$ of type $A$ and containing one of the two support; there exists such a component because we are in finite type. Assume $\alpha$ is the root whose support is contained in $I^{\prime}$ (the other case is identical). We will proceed by induction on the cardinality of $I^{\prime}$. 
Let $j$ be the only vertex in $\operatorname{Supp}(\alpha)$ connected to $i$. Without loss of generality we can assume $j \prec_{c} i$, i.e., $s_{j}$ precedes $s_{i}$ in any reduced expression of $c$. If this is not the case we can use Proposition 6 since two roots are spaced if and only if their images under the involution $\delta \mapsto \bar{\delta}$ are spaced.

Apply $\tau_{c}^{-1}$ to both $\alpha$ and $\gamma$; they are positive so $\tau_{c}^{-1}$ acts as $c^{-1}$ on them. By construction we have

$$
\operatorname{Supp}\left(\tau_{c}^{-1} \alpha\right) \subseteq I^{\prime} \backslash\{j\}
$$

and

$$
\operatorname{Supp}\left(\tau_{c}^{-1} \gamma\right) \subseteq\left(I \backslash I^{\prime}\right) \cup\{i\}
$$

where both relations hold since $s_{j}$ is applied before $s_{i}$ and $\alpha$ belongs to a type $A$ component of $I$. If one among $\tau_{c}^{-1} \alpha$ and $\tau_{c}^{-1} \gamma$ is negative we are done (again using Lemma 2 if needed) otherwise the statement follows by induction on $\left|I^{\prime}\right|$.

To complete the proof of Proposition 5 we need to sharpen Lemma 1. From this moment on we will denote a word on the alphabet $\left\{s_{i}\right\}_{i \in I}$ (up to commutations) by $\mathbf{w}$; the corresponding element in $W$ will be denoted by $w$. For convenience we will record a sequence of elementary moves by the corresponding word. As an example in type $A_{4}$ (again using the standard numeration of simple roots from [1]) the sequence of elementary moves

$$
s_{1} s_{2} s_{3} s_{4} \rightarrow s_{2} s_{3} s_{4} s_{1} \rightarrow s_{1} s_{3} s_{4} s_{2}
$$

will be encoded by $\mathbf{w}=s_{2} s_{1}$; indeed

$$
\left(s_{2} s_{1}\right)\left(s_{1} s_{2} s_{3} s_{4}\right)\left(s_{2} s_{1}\right)^{-1}=s_{1} s_{3} s_{4} s_{2} .
$$

The key observation is given by the following Lemma.

Lemma 3 For any pair of Coxeter elements $c$ and $c^{\prime}$ and for any $i \in I$, there exists a sequence of elementary moves connecting $c$ and $c^{\prime}$ that does not contain $s_{i}$.

Proof The result is obvious once we notice that both the sequences of simple moves $\mathbf{w}=s_{1}$ and $\mathbf{w}^{\prime}=s_{2} \cdots s_{n}$ acts in the same way on $c=s_{1} \cdots s_{n}$.

The Dynkin diagram $I$ is in general a forest. For any leaf $i$ in $I$, i.e., for any node belonging to a single edge, denote by $i_{\#}$ the only other node of $I$ connected to $i$.

Lemma 4 For any leaf $i \in I$ and for any Coxeter element $c$ there exist a bipartite Coxeter element $t$ and a sequence of elementary moves $\mathbf{w}$ such that

1. $c=\mathbf{w} t \mathbf{w}^{-1}$

2. $\mathbf{w}$ contains neither $s_{i}$, nor $s_{i_{\#}}$.

Proof According to Lemma 3 we can find a sequence of elementary moves $\mathbf{w}$ not containing $s_{i_{\#}}$ that transform $c$ into either of the bipartite Coxeter elements. By construction $s_{i}$ commutes with all reflections appearing in $\mathbf{w}$ since it commutes with all 
the simple reflections except $s_{i \#}$. Therefore, using commutations relations, we can always find such a w containing at most one copy of $s_{i}$. Choosing now a bipartite Coxeter element $t$ in which $s_{i}$ and $s_{i_{\#}}$ appear in the same order in which they appear in $c$ we see that $\mathbf{w}$ does not contain $s_{i}$.

Let $i$ be a leaf of $I$ and $c$ be any Coxeter element. Let $t$ and $\mathbf{w}$ be, respectively, the bipartite Coxeter element and the sequence of elementary moves constructed in Lemma 4. To fix ideas suppose that $s_{i}$ appears on the left of $s_{i \#}$ in $c$ (and in $t$ ); the other case can be dealt with exactly in the same way but multiplying on the right instead of on the left. Denote by $c_{J}$ and $t_{J}$ the corresponding Coxeter elements for the Dynkin sub-diagram $J=I \backslash\{i\}$. By our assumption $c_{J}=s_{i} c$ and $t_{J}=s_{i} t$.

Lemma 5 In the notation just established $\mathbf{w}$ is a sequence of elementary moves in $W_{J}$ conjugating $t_{J}$ and $c_{J}$.

Proof

$$
c_{J}=s_{i} c=s_{i} \mathbf{w} t \mathbf{w}^{-1}=\mathbf{w} s_{i} t \mathbf{w}^{-1}=\mathbf{w} t_{J} \mathbf{w}^{-1} .
$$

We now have all the required tools to prove Proposition 5.

Proof (Proposition 5) We can assume, without loss of generality, I to be connected. It suffices to show that the result holds when $J$ is obtained from $I$ removing one node $i$. Let $\alpha$ and $\gamma$ be roots in $\Phi_{\text {ap }}\left(c_{J}\right)$. There are two cases to consider depending on the relative position of $\operatorname{Supp}(\alpha), \operatorname{Supp}(\gamma)$ and $i$.

1. If $\operatorname{Supp}(\alpha)$ and $\operatorname{Supp}(\gamma)$ belong to different connected components of $J$ then

$$
(\alpha \| \gamma)_{c_{J}}^{J}=0=(\iota(\alpha) \| \iota(\gamma))_{c} .
$$

The first equality holds because of Remark 10 and the second one is an instance of Proposition 13.

2. If $\operatorname{Supp}(\alpha)$ and $\operatorname{Supp}(\gamma)$ belong to the same connected component of the Dynkin diagram $J$ then we can assume $i$ to be a leaf of $I$. Let $i_{\#}$ be the only vertex in $I$ connected to $i$. By Lemmata 4 and 5, there exist a sequence of elementary moves $\mathbf{w}$ and a bipartite Coxeter element $t$ such that

$$
\begin{gathered}
c=\mathbf{w} t \mathbf{w}^{-1} \\
c_{J}=\mathbf{w} t_{J} \mathbf{w}^{-1}
\end{gathered}
$$

and $\mathbf{w}$ contains neither $s_{i}$, nor $s_{i \# \text { }}$. Denote by $\sigma_{\mathbf{w}}$ the composition of the maps $\sigma_{i}$ corresponding to $\mathbf{w}$. By construction neither of $\sigma_{\mathbf{w}} \alpha$ and $\sigma_{\mathbf{w}} \gamma$ contains $i$ in its support. Using point 2 of Corollary 2 we can conclude

$$
(\alpha \| \gamma)_{c}=\left(\sigma_{\mathbf{w}} \alpha \| \sigma_{\mathbf{w}} \gamma\right)_{t}=\left(\sigma_{\mathbf{w}} \alpha \| \sigma_{\mathbf{w}} \gamma\right)_{t_{J}}^{J}=(\alpha \| \gamma)_{c_{J}}^{J} .
$$




\section{Proof of the main results}

To prove Theorem 7 we will use the following easy observation.

Lemma 6 Let $J \subset I$ be a Dynkin sub-diagram. There is a bijection between $c$ clusters in $\Phi_{\mathrm{ap}}(c)$ containing $\left\{-\beta_{i}^{c}\right\}_{i \in I \backslash J}$ and $c_{J}$-clusters in $\Phi_{\mathrm{ap}}^{J}\left(c_{J}\right)$.

The existence of such a bijection is a direct consequence of the fact that if $-\beta_{i}^{c}$ is in a $c$-cluster $C$, then for any other root $\gamma$ in that $c$-cluster $i \notin \operatorname{Supp}(\gamma)$. In particular any positive root in $C$ is contained in a complement of the space generated by

$$
\left\{-\beta_{i}^{c}\right\}_{-\beta_{i}^{c} \in C \cap \Phi_{-}}
$$

Definition 5 Call a $c$-cluster $C$ in $\Phi_{\text {ap }}(c)$ positive if $C \subset \Phi_{+}$.

Proof (Theorem 7) As already mentioned in Remark 5, the set

$$
\left\{-\beta_{i}^{c}\right\}_{i \in I}
$$

is a $\mathbb{Z}$-basis of $Q$. By Lemma 6 it suffices to show that the theorem holds for any given positive cluster $C$. Apply $\tau_{c}^{-1}$ to $C$; since it is positive $\tau_{c}^{-1}$ acts on all the roots in it as $c^{-1}$. Since $c^{-1}$ is a product of reflections, $C$ is a $\mathbb{Z}$-basis if and only if $\tau_{c}^{-1} C$ is a $\mathbb{Z}$-basis. Continue to apply $\tau_{c}^{-1}$ until one of the roots is sent to a negative one. This will happen because, similarly to the case of $\Pi(c)$, any $\tau_{c}$-orbit contains precisely two negative roots $\left\{-\beta_{i}^{c},-\beta_{i^{*}}^{c}\right\}$ or a single negative root $-\beta_{i}^{c}$ if $i=i^{*}$. Remove the negative root just obtained again using Lemma 6 and conclude by induction on the rank of the root system.

Remark 12 The proof just proposed is a straightforward adaptation of the proof of Theorem 1.8 in [6] to the new setup of $c$-almost positive roots.

Proof (Proposition 4) Let $c=s_{1} \ldots s_{n}$; for $\gamma \in Q$ write

$$
\gamma=-\sum_{i \in I} m_{-\beta_{i}^{c}} \beta_{i}^{c}+\gamma_{+}
$$

where the coefficients $m_{-} \beta_{i}^{c}$ are the non-negative integers uniquely defined (since the change of basis $\alpha_{i} \mapsto \beta_{i}^{c}$ is triangular) by the recursive formula

$$
m_{-\beta_{i}^{c}}:=\left[-\gamma-\sum_{j=1}^{i-1} m_{-\beta_{j}^{c}} \beta_{j}^{c} ; \alpha_{n}\right]_{+}
$$

(we use the convention that the empty sum is 0 ). By construction $\gamma_{+}$, the positive part of $\gamma$, is in the positive cone of the sub-root lattice generated by

$$
\left\{\alpha_{i} \mid m_{-\beta_{i}^{c}}=0\right\}
$$


Clearly $\gamma \in Q$ has a unique $c$-cluster expansion if and only if $\gamma_{+}$does. Without loss of generality we can thus assume that $\gamma$ is in the positive cone $Q_{+}$.

The root $-\beta_{i}^{c}$ can appear with a positive coefficient in a $c$-cluster expansion of $\gamma$ only if the coefficient $\left[\gamma ; \alpha_{i}\right]$ is negative therefore the result holds for $\gamma=0$ and we can assume $\gamma \neq 0$.

If $\sum_{\alpha \in \Phi_{+}} m_{\alpha} \alpha$ is a $c$-cluster expansion of $\gamma$ then

$$
\tau_{c}^{-1} \gamma=c^{-1} \gamma=c^{-1}\left(\sum_{\alpha \in \Phi_{+}} m_{\alpha} \alpha\right)=\sum_{\alpha \in \Phi_{+}} m_{\alpha} c^{-1} \alpha=\sum_{\alpha \in \Phi_{+}} m_{\alpha} \tau_{c^{-1}} \alpha
$$

is a $c$-cluster expansion of $\tau_{c}^{-1} \gamma$. In other words $\gamma$ has a unique $c$-cluster expansion if and only if $\tau_{c}^{-1} \gamma$ does. Applying $\tau_{c}^{-1}$ a sufficient number of times $\gamma$ can be moved outside of the positive cone. We can then take its positive part and conclude by induction on the rank of the root system.

Remark 13 This proof, as its analog in [6], has the advantage of considering one Coxeter element at a time. An alternative strategy could have been the following. The claim holds for bipartite Coxeter elements by point 4 in Corollary 2. Using the fact that the maps $\sigma_{i}$ preserve compatibility degree one can then transfer the property to other sets of $c$-almost positive roots.

As in [6], Theorem 8 follows from Proposition 4. For the sake of completeness we replicate the proof here.

Proof (Theorem 8) It suffices to show that

1. no two cones of $\mathcal{F}_{c}^{\Phi}$ have a common interior point

2. the union of all cones is $Q_{\mathbb{R}}$.

Assume by contradiction that there exists a point in the common interior of two cones. Since $Q_{\mathbb{Q}}$ is dense in $Q_{\mathbb{R}}$ we may assume that such point is in $Q_{\mathbb{Q}}$. Clearing the denominators there is then a common point in $Q$ which contradicts the uniqueness of the $c$-cluster expansion. Therefore the interiors of any two cones are disjoint.

Since any $\gamma \in Q$ has a $c$-cluster expansion the union of all the cones $\mathbb{R}_{+} C$ contains $Q$; since this union is closed in $Q_{\mathbb{R}}$ and stable under the action of $\mathbb{R}_{+}$it must contain all of $Q_{\mathbb{R}}$ and we are done.

To prove Theorem 9 we will apply the criterion provided by Lemma 2.1 in [3]. Let us restate it in the particular case we need.

Lemma 7 Let $F_{c}$ be a continuous piecewise-linear function

$$
F_{c}: Q_{\mathbb{R}} \longrightarrow \mathbb{R}
$$

linear on the maximal cones of the fan $\mathcal{F}_{c}^{\Phi}$ (as such $F_{c}$ is uniquely determined by its values on $\Phi_{\text {ap }}(c)$ ). Then $\mathcal{F}_{c}^{\Phi}$ is the normal fan to a unique full-dimensional polytope with support function $F_{c}$ if and only if $F_{c}$ satisfy the following system of inequalities. 
For any pair of adjacent c-clusters $C_{\alpha}$ and $C_{\gamma}$ let $\alpha$ be the only root in $C_{\alpha} \backslash C_{\gamma}$ and $\gamma$ the only root in $C_{\gamma} \backslash C_{\alpha}$. Let

$$
m_{\alpha} \alpha+m_{\gamma} \gamma=\sum_{\delta \in C_{\alpha} \cap C_{\gamma}} m_{\delta} \delta
$$

be the unique (up to non-zero scalar multiple) linear dependence on the elements of $C_{\alpha} \cup C_{\gamma}$ with $m_{\alpha}$ and $m_{\gamma}$ positive. Then

$$
m_{\alpha} F_{c}(\alpha)+m_{\gamma} F_{c}(\gamma)>\sum_{\delta \in C_{\alpha} \cap C_{\gamma}} m_{\delta} F_{c}(\delta) .
$$

In particular the domains of linearity of $F_{c}$ are exactly the maximal cones of $\mathcal{F}_{c}^{\Phi}$.

To apply Lemma 7 we make the relations (15) more explicit by exploring the interaction of $\sigma_{1}$ and $\tau_{c}$. Note that, having established Theorem 8, any vector-valued function on $\Phi_{\text {ap }}(c)$ can be extended to a continuous piecewise-linear map on $Q_{\mathbb{R}}$; in particular this is the case for $\tau_{c}$ and $\sigma_{i}$.

To avoid degenerate cases, from now on, assume that every connected component of $I$ contains at least 2 vertices. As before, let $c$ be $s_{1} \cdots s_{n}$.

Lemma 8 Let $\alpha$ and $\gamma$ be roots in $\Phi_{\mathrm{ap}}(c)$ such that

$$
(\alpha \| \gamma)_{c}=1=(\gamma \| \alpha)_{c}
$$

Then

$$
\sigma_{1}^{-1}\left(\sigma_{1}(\alpha)+\sigma_{1}(\gamma)\right)
$$

is either $\alpha+\gamma$ or

$$
\tau_{c}\left(\tau_{c}^{-1}(\alpha)+\tau_{c}^{-1}(\gamma)\right)
$$

and it is different from $\alpha+\gamma$ only if one of the two roots (say, $\alpha)$ is $-\beta_{1}^{c}$; in this case

$$
\sigma_{1}^{-1}\left(\sigma_{1}\left(-\beta_{1}^{c}\right)+\sigma_{1}(\gamma)\right)=\gamma-\alpha_{1} \in Q_{+}
$$

Proof If both $\alpha$ and $\gamma$ are positive roots then

$$
\sigma_{1}^{-1}\left(\sigma_{1}(\alpha)+\sigma_{1}(\gamma)\right)=\sigma_{1}^{-1}\left(s_{1} \alpha+s_{1} \gamma\right)=\sigma_{1}^{-1}\left(s_{1}(\alpha+\gamma)\right)
$$

Let

$$
\alpha+\gamma=\sum_{\delta \in \Phi_{\mathrm{ap}}(c)} m_{\delta} \delta
$$

be the $c$-cluster expansion of $\alpha+\gamma$; all the roots $\delta$ such that $m_{\delta} \neq 0$ are positive; therefore

$$
\sigma_{1}^{-1}\left(s_{1}(\alpha+\gamma)\right)=\sigma_{1}^{-1}\left(s_{1}\left(\sum m_{\delta} \delta\right)\right)=\sigma_{1}^{-1}\left(\sigma_{1}(\alpha+\gamma)\right)=\alpha+\gamma
$$


It remains to consider the case in which one of the two roots is negative (they cannot be both negative); we can, by symmetry, assume $\alpha$ to be the negative one. If $\alpha=-\beta_{i}^{c}$ with $i \neq 1$ then

$$
\sigma_{1}^{-1}\left(\sigma_{1}\left(-\beta_{i}^{c}\right)+\sigma_{1}(\gamma)\right)=\sigma_{1}^{-1}\left(-s_{1} \beta_{i}^{c}+s_{1} \gamma\right)=\sigma_{1}^{-1}\left(s_{1}\left(\gamma-\beta_{i}^{c}\right)\right) .
$$

Let

$$
\gamma-\beta_{i}^{c}=\sum_{\delta \in \Phi_{\mathrm{ap}}(c)} m_{\delta} \delta
$$

be the $c$-cluster expansion of $\gamma-\beta_{i}^{c}$. None of the roots $\delta$ appearing with a positive coefficient is $-\beta_{1}^{c}$ since

$$
\left[\gamma-\beta_{i}^{c} ; \alpha_{1}\right] \geq 0
$$

( $-\beta_{1}^{c}$ is the only negative root in $\Phi_{\mathrm{ap}}(c)$ having $\alpha_{1}$ with non-zero coefficient). Therefore on $\gamma-\beta_{i}^{c}$ the actions of $s_{1}$ and of $\sigma_{1}$ are the same. We get

$$
\sigma_{1}^{-1}\left(\sigma_{1}\left(-\beta_{i}^{c}\right)+\sigma_{1}(\gamma)\right)=\sigma_{1}^{-1}\left(s_{1}\left(\gamma-\beta_{i}^{c}\right)\right)=\sigma_{1}^{-1}\left(\sigma_{1}\left(\gamma-\beta_{i}^{c}\right)\right)=\gamma-\beta_{i}^{c} .
$$

Finally if $\alpha=-\beta_{1}^{c}$ then on the one hand we have

$$
\sigma_{1}^{-1}\left(\sigma_{1}\left(-\beta_{1}^{c}\right)+\sigma(\gamma)\right)=\sigma_{1}^{-1}\left(\alpha_{1}+s_{1} \gamma\right)=\sigma_{1}^{-1}\left(s_{1}\left(\gamma-\alpha_{1}\right)\right)
$$

$\gamma-\alpha_{1}$ is in $Q_{+}$therefore

$$
\sigma_{1}^{-1}\left(s_{1}\left(\gamma-\alpha_{1}\right)\right)=\sigma_{1}^{-1}\left(\sigma_{1}\left(\gamma-\alpha_{1}\right)\right)=\gamma-\alpha_{1} .
$$

On the other hand

$$
\tau_{c}\left(\tau_{c}^{-1}\left(-\beta_{1}^{c}\right)+\tau_{c}^{-1}(\gamma)\right)=\tau_{c}\left(\beta_{1}^{c}+c^{-1} \gamma\right)=\tau_{c}\left(c^{-1}\left(\gamma-\alpha_{1}\right)\right)
$$

we can interchange $c^{-1}$ and $\tau_{c}^{-1}$ because $\gamma-\alpha_{1}$ is in $Q_{+}$and conclude

$$
\tau_{c}\left(c^{-1}\left(\gamma-\alpha_{1}\right)\right)=\tau_{c}\left(\tau_{c}^{-1}\left(\gamma-\alpha_{1}\right)\right)=\gamma-\alpha_{1} .
$$

Proposition 14 Let $\alpha$ and $\gamma$ be roots in $\Phi_{\mathrm{ap}}(c)$ such that

$$
(\alpha \| \gamma)_{c}=1=(\gamma \| \alpha)_{c} .
$$

Then

$$
\left\{\tau_{c}^{m}\left(\tau_{c}^{-m}(\alpha)+\tau_{c}^{-m}(\gamma)\right)\right\}_{m \in \mathbb{Z}}
$$

consist of exactly two elements, one is $\alpha+\gamma$; denote the other by $\alpha \uplus_{c} \gamma$.

Proof If $c$ is bipartite there is nothing to prove by point 1 in Corollary 3. In view of Lemma 8, for any other Coxeter element $c=s_{1} \cdots s_{n}$ and any integer $m$ we have

$$
\tau_{s_{1} c s_{1}}^{m}\left(\tau_{s_{1} c s_{1}}^{-m}\left(\sigma_{1}(\alpha)\right)+\tau_{s_{1} c s_{1}}^{-m}\left(\sigma_{1}(\gamma)\right)\right)=\sigma_{1} \tau_{c}^{m} \sigma_{1}^{-1}\left(\sigma_{1} \tau_{c}^{-m}(\alpha)+\sigma_{1} \tau_{c}^{-m}(\gamma)\right) .
$$


For a suitable $m^{\prime}$ in $\{m, m+1\}$ we get

$$
\sigma_{1} \tau_{c}^{m} \sigma_{1}^{-1}\left(\sigma_{1} \tau_{c}^{-m}(\alpha)+\sigma_{1} \tau_{c}^{-m}(\gamma)\right)=\sigma_{1} \tau_{c}^{m^{\prime}}\left(\tau_{c}^{-m^{\prime}}(\alpha)+\tau_{c}^{-m^{\prime}}(\gamma)\right)
$$

Therefore

$$
\left\{\sigma_{1}(\alpha)+\sigma_{1}(\gamma), \sigma_{1}(\alpha) \uplus_{s_{1} c s_{1}} \sigma_{1}(\gamma)\right\} \subseteq \sigma_{1}\left\{\alpha+\gamma, \alpha \uplus_{c} \gamma\right\}
$$

and the claim follows reversing the role of $c$ and $s_{1} c s_{1}$.

Remark 14 Following Remark 1.15 in [3], if $I$ contains a component of type $A_{1}$ let $\alpha_{1}$ and $-\alpha_{1}$ be the corresponding roots. In view of Remark 10 they are both compatible with any root in $\Phi_{\text {ap }}(c) \backslash\left\{\alpha_{1},-\alpha_{1}\right\}$. By direct inspection, we have

$$
\left(-\alpha_{1}|| \alpha_{1}\right)_{c}=1=\left(\alpha_{1} \|-\alpha_{1}\right)_{c} .
$$

In this case their sum is 0 and it is natural to declare $-\alpha_{1} \uplus_{c} \alpha_{1}$ to be 0 too.

\section{Corollary 4 If}

$$
(\alpha \| \gamma)_{c}=1=(\gamma \| \alpha)_{c}
$$

then every root appearing with positive coefficient in the cluster expansion of either $\alpha+\gamma$ or $\alpha \uplus_{c} \gamma$ is compatible with $\alpha, \gamma$ and with any other root compatible with both $\alpha$ and $\gamma$.

Proof The statement is true in the bipartite case by point 2 in Corollary 3. For an arbitrary Coxeter element $c=s_{1} \cdots s_{n}$ the result can be deduced using elementary moves: from the previous proof we have

$$
\left\{\sigma_{1}(\alpha)+\sigma_{1}(\gamma), \sigma_{1}(\alpha) \uplus_{s_{1} c s_{1}} \alpha_{1}(\gamma)\right\}=\sigma_{1}\left(\left\{\alpha+\gamma, \alpha \uplus_{c} \gamma\right\}\right)
$$

and the claim follows since $\sigma_{1}$ preserves compatibility degrees.

Lemma 9 In every dependence relation (15) we have

$$
(\alpha \| \gamma)_{c}=1=(\gamma \| \alpha)_{c}
$$

Furthermore, after normalization the relation (15) is just the c-cluster expansion of $\alpha+\gamma$ :

$$
\alpha+\gamma=\sum_{\delta \in \Phi_{\mathrm{ap}}(c)} m_{\delta} \delta
$$

Proof Normalize (15) so that coefficients are coprime integers. By Theorem 7 all the coefficients in

$$
\alpha=-\frac{m_{\gamma}}{m_{\alpha}} \gamma+\sum_{\delta \in C_{\alpha} \cap C_{\gamma}} \frac{m_{\delta}}{m_{\alpha}} \delta
$$


are integers forcing $m_{\alpha}=1$ (it is positive by hypothesis). In a similar fashion $m_{\gamma}=1$.

To show (18), using the $\tau_{c}$-invariance of the compatibility degree, it suffices to consider the case $\alpha=-\beta_{i}^{c}$. We have

$$
\gamma=\beta_{i}^{c}+\sum_{\delta \in C_{-\beta_{i}^{c}} \cap C_{\gamma}} m_{\delta} \delta
$$

and thus

$$
\left(-\beta_{i}^{c} \| \gamma\right)_{c}=\left[\gamma ; \alpha_{i}\right]_{+}=1
$$

since $i$ is not in $\operatorname{Supp}(\delta)$ for any $\delta$ in $C_{-\beta_{i}^{c}} \cap C_{\gamma}$.

The fact that, after the normalization, the dependence (15) is the $c$-cluster expansion of $\alpha+\gamma$ is a direct application of Corollary 4. Any root appearing with non-zero coefficient in the cluster expansion of $\alpha+\gamma$ is compatible with $\alpha, \gamma$, and with any other root compatible with both $\alpha$ and $\gamma$, therefore it is a root in $C_{\alpha} \cap C_{\gamma}$.

Proposition 14 together with Corollary 4 and Lemma 9 allow us to compute exchange relations. Let $\mathcal{A}_{0}(c)$ be the coefficient-free cluster algebra with initial exchange matrix $B(c)$ and denote by $\left\{x_{\alpha, c}\right\}_{\alpha \in \Phi_{\text {ap }}(c)}$ its cluster variables. Due to Proposition 4 all the cluster monomials are in bijection with points of $Q$. Namely we can write

$$
x_{\gamma, c}:=\prod_{\delta \in \Phi_{\mathrm{ap}}(c)} x_{\delta, c}^{m_{\delta}}
$$

where

$$
\gamma=\sum_{\delta \in \Phi_{\mathrm{ap}}(c)} m_{\delta} \delta
$$

is the cluster expansion of $\gamma \in Q$.

Proof (Theorem 10) The statement is true when $c$ is a bipartite Coxeter element (cf. (5.1) in [5]). Let $c=s_{1} \cdots s_{n}$. We have

$$
\begin{aligned}
x_{\alpha, s_{1} c s_{1}} x_{\gamma, s_{1} c s_{1}} & =x_{\sigma_{1}^{-1}(\alpha), c} x_{\sigma_{1}^{-1}(\gamma), c} \\
& =x_{\sigma_{1}^{-1}(\alpha)+\sigma_{1}^{-1}(\gamma), c}+x_{\sigma_{1}^{-1}(\alpha) \uplus_{c} \sigma_{1}^{-1}(\gamma), c} \\
& =x_{\sigma_{1}\left(\sigma_{1}^{-1}(\alpha)+\sigma_{1}^{-1}(\gamma)\right), s_{1} c s_{1}}+x_{\sigma_{1}\left(\sigma_{1}^{-1}(\alpha) \uplus_{c} \sigma_{1}^{-1}(\gamma)\right), s_{1} c s_{1}} \\
& =x_{\alpha+\gamma, s_{1} c s_{1}}+x_{\alpha \uplus_{s_{1} c s_{1}} \gamma, s_{1} c s_{1}} .
\end{aligned}
$$

Recall Remark 6: by construction of the map $\tau_{c}$, there is one $\tau_{c}$-orbit in $\Phi_{\text {ap }}(c)$ for each $w_{0}$-orbit in $I$, i.e., there exists $-\beta_{j}^{c}$ such that

$$
\tau_{c}^{m}\left(-\beta_{i}^{c}\right)=-\beta_{j}^{c}
$$

if and only if $j \in\left\{i, i^{*}\right\}$. 
Since $\sigma_{j}$ sends $-\beta_{i}^{c}$ to $\left\{ \pm \beta_{i}^{s_{j} c s_{j}}\right\}$ the $\tau_{c}$-orbit of $-\beta_{i}^{c}$ gets mapped to the $\tau_{s_{j} c s_{j}}$ orbit of $-\beta_{i}^{s_{j} c s_{j}}$. In particular, for any function

$$
f: I \longrightarrow \mathbb{R}
$$

such that

$$
f(i)=f\left(i^{*}\right)
$$

we get a family of maps, one for each Coxeter element $c$,

$$
F_{c}=F_{c ; f}: \Phi_{\mathrm{ap}}(c) \longrightarrow \mathbb{R}
$$

defined setting $F_{c}\left(-\beta_{i}^{c}\right):=f(i)$ and extending by $\tau_{c}$-invariance. These maps are invariant under the action of $\sigma_{i}$, that is

$$
F_{s_{i} c s_{i}}\left(\sigma_{i}(\alpha)\right)=F_{c}(\alpha)
$$

for any $c$, any $i$ initial in $c$, and any $\alpha$ in $\Phi_{\text {ap }}(c)$. From now on assume that $F_{c}$ has been defined in this way and extend it to a continuous, piecewise-linear function

$$
F_{c}: Q_{\mathbb{R}} \longrightarrow \mathbb{R}
$$

linear on maximal cones of $\mathcal{F}_{c}$.

Proposition 15 Fix any function

$$
f: I \longrightarrow \mathbb{R}
$$

such that

1. for any $i \in I$

$$
f(i)=f\left(i^{*}\right)
$$

2. for any $j \in I$

$$
\sum_{i \in I} a_{i j} f(i)>0 .
$$

Then for any pair of roots $\alpha$ and $\gamma$ in $\Phi_{\mathrm{ap}}(c)$ such that

$$
(\alpha \| \gamma)_{c}=1=(\gamma \| \alpha)_{c}
$$

the following inequality holds:

$$
F_{c}(\alpha)+F_{c}(\gamma)>\max \left\{F_{c}(\alpha+\gamma), F_{c}\left(\alpha \uplus_{c} \gamma\right)\right\} .
$$

Proof The bipartite case was taken care of by point 3 in Corollary 3. Let $c=s_{1} \cdots s_{n}$ be any Coxeter element. Using elementary moves, (17) and (19) we get 


$$
\begin{aligned}
& F_{s_{1} c s_{1}}\left(\sigma_{1}(\alpha)\right)+F_{s_{1} c s_{1}}\left(\sigma_{1}(\gamma)\right) \\
& \quad=F_{c}(\alpha)+F_{c}(\gamma) \\
& \quad>\max \left\{F_{c}(\alpha+\gamma), F_{c}\left(\alpha \uplus_{c} \gamma\right)\right\} \\
& \quad=\max \left\{F_{s_{1} c s_{1}}\left(\sigma_{1}(\alpha+\gamma)\right), F_{s_{1} c s_{1}}\left(\sigma_{1}\left(\alpha \uplus_{c} \gamma\right)\right)\right\} \\
& \quad=\max \left\{F_{s_{1} c s_{1}}\left(\sigma_{1}(\alpha)+\sigma_{1}(\gamma)\right), F_{s_{1} c s_{1}}\left(\sigma_{1}(\alpha) \uplus_{s_{1} c s_{1}} \sigma_{1}(\gamma)\right)\right\}
\end{aligned}
$$

as desired.

Proof (Theorem 9) It is enough to note that the Proposition 15 together with Lemma 9 satisfy the requirements of Lemma 7.

\section{Relation between $\mathcal{F}_{c}^{\Pi}$ and the $c$-Cambrian fan $\mathcal{F}_{c}^{C}$}

We start by recalling some results and terminology from [11].

Definition 6 (cf. Proposition 1.1 in [11]) Fix a Coxeter element $c$ and call an element $w \in W$ a $c$-singleton if $w$ is both $c$-sortable and $c$-antisortable.

Note that both $w_{0}$ and the identity element of $W$ are $c$-singletons for any choice of $c$. Denote by $\mathbf{w}_{0}$ the $c$-sorting word of $w_{0}$.

Theorem 11 (cf. Theorem 1.2 in [11]) An element $w \in W$ is a c-singleton if and only if it has a reduced expression which is a prefix of $\mathbf{w}_{0}$ up to commutations.

Theorem 12 (cf. Theorem 2.6 in [11]) For any ray $\rho$ of $\mathcal{F}_{c}^{C}$, there exist a unique fundamental weight $\omega_{i}$ and a (non unique) c-singleton $w$ such that

$$
\rho=\mathbb{R}_{+} \cdot w \omega_{i}
$$

Conversely for any c-singleton $w$ and any fundamental weight $\omega_{i}$, the weight $w \omega_{i}$, lies on a ray of $\mathcal{F}_{c}^{C}$.

We will use Theorems 11 and 12 to relate the rays of $\mathcal{F}_{c}^{C}$ to the elements of the set $\Pi(c)$.

Definition 7 Given a Coxeter element $c \in W$, we call a reduced expression $c=$ $s_{1} \cdots s_{n}$ greedy if

$$
h(i, c) \geq h(j, c)
$$

whenever $i<j$.

Lemma 10 Any Coxeter element c admits a greedy reduced expression. 
Proof Consider a reduced expression $s_{1} \cdots s_{n}$ for $c$ and suppose $h(i, c)<h(i+1, c)$ for some $i$. Let $i$ be the minimal index with this property. Using Proposition 1.6 in [18] we can deduce that $i$ and $i+1$ are not connected in the Coxeter graph. Indeed if they were connected then we would have $i \prec_{c} i+1$ and thus $h(i, c) \geq h(i+1, c)$ which is in contradiction with our assumption. Therefore $s_{i}$ and $s_{i+1}$ commute and $s_{1} \cdots s_{i+1} s_{i} \cdots s_{n}$ is another reduced expression for $c$; we can now conclude by induction.

Remark 15 Greedy reduced expressions, in general, are not unique; for example $s_{2} s_{4} s_{1} s_{3}$ and $s_{4} s_{2} s_{1} s_{3}$ are both greedy reduced expression of the same Coxeter element in type $A_{4}$ (again we used the standard numeration of roots from [1]).

Lemma 11 For any vertices $i$ and $j$ of the Dynkin diagram at distance $d$ from each other the difference $h(i, c)-h(j, c)$ is at most $d$.

Proof It is enough to observe that if $i$ and $j$ are adjacent then either $i \prec_{c} j$ or $j \prec_{c} i$ so $|h(i, c)-h(j, c)| \leq 1$ by Proposition 1.6 in [18]. Therefore each step on the minimal path in $I$ connecting $i$ and $j$ contribute at most 1 to the difference $h(i, c)-h(j, c)$.

Fix a greedy reduced expression for $c$. With some abuse of notation, we denote this expression also as $c$. Denote by $\mathbf{w}_{m}$ the sub-word of $c^{m}$ obtained by omitting in the $l$ th copy of $c$ all the transpositions $s_{i}$ such that $h(i, c)<l$. Observe that having taken a greedy reduced expression for $c$, if we write $I_{1}, \ldots I_{m}$ for the $c$-factorization of $\mathbf{w}_{m}$, then

$$
I_{1} \supseteq I_{2} \cdots \supseteq I_{m}
$$

In particular if $\mathbf{w}_{m}$ is a reduced word then $w_{m}$, the corresponding element of $W$, is $c$-sortable. Let $m_{c}=\max _{i \in I}\{h(i, c)\}$, our goal is to show that the word $\mathbf{w}_{m_{c}}$ is a reduced expression for $w_{0}$.

Proposition 16 For any $i \in I$ and any $m \leq h(i, c)$ we have $c^{m} \omega_{i}=\mathbf{w}_{m} \omega_{i}$.

Proof Let $I_{1}, \ldots, I_{m}$ be the $c$-factorization of $\mathbf{w}_{m}$ with respect to the fixed greedy reduced expression of $c$. Observe that, for any $j$ appearing in $I_{l+1}$ and for any $k$ missing from $I_{l}$,

$$
|h(k, c)-h(j, c)| \geq 2
$$

and so, by Lemma $11, s_{k}$ and $s_{j}$ commute. Consider now the element

$$
\mathbf{W}=c_{I \backslash I_{1}} c_{I \backslash I_{2}} \cdots c_{I \backslash I_{m}} .
$$

Since $m \leq h(i, c)$, the reflection $s_{i}$ will not appear in $\mathbf{w}$ and so $\mathbf{w} \omega_{i}=\omega_{i}$ hence $\mathbf{w}_{m} \mathbf{w} \omega_{i}=\mathbf{w}_{m} \omega_{i}$. Form the previous consideration we can move all the elements in the $l$ th copy of $c$ in $\mathbf{w}$ up to the $l$ th block of $\mathbf{w}_{m}$ and obtain

$$
c^{m} \omega_{i}=c_{I_{1}} c_{I \backslash I_{1}} \cdots c_{I_{m}} c_{I \backslash I_{m}} \omega_{i}=\mathbf{w}_{m} \mathbf{w} \omega_{i}=\mathbf{w}_{m} \omega_{i} .
$$


Proposition $17 \mathbf{w}_{m_{c}}$ is a reduced expression of $w_{0}$.

Proof To show that $\mathbf{w}_{m_{c}}$ is an expression of $w_{0}$ it is enough to show that both $w_{0}$ and $\mathbf{w}_{m_{c}}$ act in the same way on the weight space (the representation of $W$ as reflection group of $P_{\mathbb{R}}$ is faithful). Fundamental weights form a basis of the weight space so it is enough to see how $w_{0}$ and $\mathbf{w}_{m_{c}}$ act on them. For any $i$ we have $w_{0} \omega_{i}=-\omega_{i^{*}}$. On the other hand, using Proposition 16, we conclude that

$$
\mathbf{w}_{m_{c}} \omega_{i}=\mathbf{w}_{h(i, c)} \omega_{i}=c^{h(i, c)} \omega_{i}=-\omega_{i} *
$$

Therefore $\mathbf{w}_{m_{c}}$ is a word representing $w_{0}$. The fact that it is a reduced expression follows from considerations on its length; each reflection $s_{i}$ appears exactly $h(i, c)$ times in it. Proposition 1.7 in [18] states that, for every $i$, the $\operatorname{sum} h(i, c)+h\left(i^{*}, c\right)$ is equal to the Coxeter number $h$, hence

$$
\sum_{i \in I}\left(h(i, c)+h\left(i^{*}, c\right)\right)=|I| h=|\Phi|
$$

but in this way we are counting the contribution of each $i$ twice, i.e.

$$
l\left(\mathbf{w}_{m_{c}}\right) \leq \sum_{i \in I} h(i, c)=\frac{1}{2} \sum_{i \in I}\left(h(i, c)+h\left(i^{*}, c\right)\right)=\frac{1}{2}|\Phi|=\left|\Phi_{+}\right|=l\left(w_{0}\right) .
$$

Note that, in view of last Proposition, for any $m \leq m_{c}, \mathbf{w}_{m}$ is a reduced expression in $W$ (and $w_{m}$ is $c$-sortable).

Proposition 18 Fix a greedy reduced expression for $c$. Then $\mathbf{w}_{m_{c}}$ is the lexicographically first reduced expression of $w_{0}$ as a sub-word of $c^{\infty}$. In other words $\mathbf{w}_{m_{c}}$ is the $c$-sorting word of $w_{0}$.

Proof It is enough to show that $w_{m} \alpha_{i}$ is a negative root for any $i$ not in $I_{m}$. We have

$$
0<\left(\alpha_{i}, \omega_{i}\right)=\left(w_{m} \alpha_{i}, w_{m} \omega_{i}\right)=\left(w_{m} \alpha_{i}, w_{0} \omega_{i}\right)=\left(w_{m} \alpha_{i},-\omega_{i} *\right)
$$

thus $\left(w_{m} \alpha_{i}, \omega_{i} *\right)<0$ and so $w_{m} \alpha_{i}$ is a negative root.

Remark 16 Combining together Theorem 11 and Proposition 18 we get another characterization of $c$-singletons: they are all the prefixes of $\mathbf{w}_{m_{c}}$ up to commutations.

Proposition 19 The sets of rays of $\mathcal{F}_{c}^{\Pi}$ and $\mathcal{F}_{c}^{C}$ coincide.

Proof Fix a greedy reduced expression for $c$. Let $\rho$ be a ray of $\mathcal{F}_{c}^{C}$. By Theorem 12 there exist a $c$-singleton $w$ and a fundamental weight $\omega_{i}$ such that

$$
\rho=\mathbb{R}_{+} w \omega_{i} .
$$

Let $m$ be the minimum integer such that $w$ is a prefix of $\mathbf{w}_{m}$. By Proposition 16

$$
w \omega_{i}=\mathbf{w}_{m} \omega_{i}=c^{m} \omega_{i} \in \Pi(c) .
$$


On the other hand, given any element $c^{m} \omega_{i}$ of $\Pi(c)$, let $\mathbf{w}_{m}$ be the corresponding sub-word of $c^{m}$ as in Proposition 16; it is a $c$-singleton therefore $c^{m} \omega_{i}=\mathbf{w}_{m} \omega_{i}$ is a point on a ray of $\mathcal{F}_{c}^{C}$ by Theorem 12 .

We can now define the polytope $\operatorname{Asso}_{c}^{a}(W)$. For any point $a$ in $P_{\mathbb{R}}$ and for any ray $\rho$ of $\mathcal{F}_{c}^{C}$ such that $\rho=\mathbb{R}_{+} \cdot w \omega_{j}$, denote by $\mathcal{H}_{\rho}^{a}$ the half-space

$$
\mathcal{H}_{\rho}^{a}:=\left\{\varphi \in P_{\mathbb{R}}^{*} \mid \varphi\left(w \omega_{j}\right) \leq\left(a, \omega_{j}\right)\right\} .
$$

The main result in [11] is that, if $a$ lies in the interior of the fundamental Weyl chamber, the intersection of half-spaces

$$
\operatorname{Asso}_{c}^{a}(W):=\bigcap \mathcal{H}_{\rho}^{a}
$$

as $\rho$ runs over all rays of $\mathcal{F}_{c}^{C}$ is a simple polytope and its normal fan is $\mathcal{F}_{c}^{C}$.

Proof (Theorem 6) In view of Proposition 19, the two polytopes become

$$
\operatorname{Asso}_{c}^{a}(W)=\left\{\varphi \in P_{\mathbb{R}}^{*} \mid \varphi\left(c^{m} \omega_{i}\right) \leq\left(a, \omega_{i}\right) \forall i \in I, 0 \leq m \leq h(i, c)\right\}
$$

and

$$
\operatorname{Asso}_{c}^{f}(W)=\left\{\varphi \in P_{\mathbb{R}}^{*} \mid \varphi\left(c^{m} \omega_{i}\right) \leq f(i) \forall i \in I, 0 \leq m \leq h(i, c)\right\} .
$$

For any function $f: I \longrightarrow \mathbb{R}$ let $a$ be the point in $P_{\mathbb{R}}$ defined by the conditions

$$
\left(a, \omega_{i}\right):=f(i)
$$

for all $i \in I$. Imposing condition 2 of Theorem 4 on $f$ is equivalent to ask for $a$ to lie in the fundamental Weyl chamber; indeed $a$ is in it if and only if the scalar product $\left(\alpha_{j}, a\right)$ is positive for every $j \in I$. Since $\alpha_{j}=\sum_{i \in I} a_{i j} \omega_{i}$ we have

$$
\left(\alpha_{j}, a\right)=\left(\sum_{i \in I} a_{i j} \omega_{i}, a\right)=\sum_{i \in I} a_{i j}\left(a, \omega_{i}\right)=\sum_{i \in I} a_{i j} f(i)>0 .
$$

We can thus conclude that, for any function $f: I \longrightarrow \mathbb{R}$ satisfying conditions 1 and 2 of Theorem 4, choosing $a$ as above, we get

$$
\operatorname{Asso}_{c}^{a}(W)=\operatorname{Asso}_{c}^{f}(W) .
$$

Remark 17 It is clear that, imposing condition 1 of Theorem 4, from our construction we get only the polytopes from [11] obtained from points $a$ invariant under the action of $-w_{0}$.

Acknowledgements I would like to thank Andrei Zelevinsky for his guidance through the work on this paper and for having introduced me to the theory of cluster algebras. Extremely helpful was the suggestion to consider the negative roots $-\beta_{i}^{c}$ instead of negative simples made by Hugh Thomas. I am grateful to Sachin Gautam for the tireless way he provided insights and the many useful conversations we had. A special thank to Nathan Reading for sharing his software and to Gregg Musiker for explaining me the 
add-on to SAGE [17] he is writing: both tools helped when producing examples. I am also grateful to Elena Collina, Andrea Appel, Giorgia Fortuna, Andrew Carrol, Federico Galetto, Shih-Wey Yang, Daniele Valeri, and Alessandro D'Andrea for listening in moments I needed to express my doubts to someone.

The author is partially supported by A. Zelevinsky's NSF grants DMS-0801187 and DMS-1103813.

\section{References}

1. Bourbaki, N.: Éléments de Mathématique. Fasc. XXXIV. Groupes et Algèbres de Lie. Chapitre IV: Groupes de Coxeter et Systèmes de Tits. Chapitre V: Groupes Engendrés Par des Réflexions. Chapitre VI: Systèmes de Racines. Actualités Scientifiques et Industrielles, vol. 1337. Hermann, Paris (1968)

2. Ceballos, C., Santos, F., Ziegler, G.M.: Many non-equivalent realizations of the associahedron. Preprint (2011). http://arxiv.org/abs/1109.5544

3. Chapoton, F., Fomin, S., Zelevinsky, A.: Polytopal realizations of generalized associahedra. Can. Math. Bull. 45(4), 537-566 (2002). Dedicated to Robert V. Moody. http://journals.cms.math.ca/ ams/ams-redirect.php?Journal $=$ CMB $\&$ Volume $=45 \&$ FirstPage $=537$

4. Derksen, H., Weyman, J., Zelevinsky, A.: Quivers with potentials and their representations II: applications to cluster algebras. J. Am. Math. Soc. 23(3), 749-790 (2010). doi:10.1090/ S0894-0347-10-00662-4

5. Fomin, S., Zelevinsky, A.: Cluster algebras. II. Finite type classification. Invent. Math. 154(1), 63-121 (2003). doi:10.1007/s00222-003-0302-y

6. Fomin, S., Zelevinsky, A.: $Y$-systems and generalized associahedra. Ann. Math. 158(3), 977-1018 (2003). doi:10.4007/annals.2003.158.977

7. Fomin, S., Zelevinsky, A.: Cluster algebras. IV. Coefficients. Compos. Math. 143(1), 112-164 (2007). doi:10.1112/S0010437X06002521

8. Fulton, W.: Introduction to Toric Varieties. Annals of Mathematics Studies, vol. 131. Princeton University Press, Princeton (1993). The William H. Roever Lectures in Geometry

9. Geck, M., Pfeiffer, G.: Characters of Finite Coxeter Groups and Iwahori-Hecke Algebras. London Mathematical Society Monographs. New Series, vol. 21. The Clarendon Press Oxford University Press, New York (2000)

10. Hohlweg, C.: Permutahedra and associahedra: generalized associahedra from the geometry of finite reflection groups. In: Mueller-Hoissen, F., Pallo, J.M., Stasheff, J. (eds.) Associahedra, Tamari Lattices and Related Structures. Birkhäuser, Basel (2012)

11. Hohlweg, C., Lange, C.E.M.C., Thomas, H.: Permutahedra and generalized associahedra. Adv. Math. 226(1), 608-640 (2011). doi:10.1016/j.aim.2010.07.005

12. Postnikov, A.: Permutohedra, associahedra, and beyond. Int. Math. Res. Not., 1026-1106 (2009)

13. Reading, N.: Lattice congruences, fans and Hopf algebras. J. Comb. Theory, Ser. A 110(2), 237-273 (2005). doi:10.1016/j.jcta.2004.11.001

14. Reading, N.: Cambrian lattices. Adv. Math. 205(2), 313-353 (2006). doi:10.1016/j.aim.2005.07.010

15. Reading, N.: Sortable elements and Cambrian lattices. Algebra Univers. 56(3-4), 411-437 (2007). doi:10.1007/s00012-007-2009-1

16. Reading, N., Speyer, D.E.: Cambrian fans. J. Eur. Math. Soc. 11(2), 407-447 (2009). doi:10.4171/ JEMS/155

17. Stein, W., et al.: Sage Mathematics Software (Version 4.7). The Sage Development Team (2011). http://www.sagemath.org

18. Yang, S.W., Zelevinsky, A.: Cluster algebras of finite type via Coxeter elements and principal minors. Transform. Groups 13(3-4), 855-895 (2008). doi:10.1007/s00031-008-9025-X 